\title{
Boundary Conditions for Sturm-Liouville Equation with Transition Regions and Barriers or Wells
}

\author{
Alfred Wünsche \\ Institut für Physik, Humboldt-Universität, Nonclassical Radiation, Berlin, Germany \\ Email: alfred.wuensche@physik.hu-berlin.de
}

How to cite this paper: Wünsche, A. (2021) Boundary Conditions for Sturm-Liouville Equation with Transition Regions and Barriers or Wells. Advances in Pure Mathematics, 11, 254-295.

https://doi.org/10.4236/apm.2021.114018

Received: February 22, 2021

Accepted: April 18, 2021

Published: April 21, 2021

Copyright $\odot 2021$ by author(s) and Scientific Research Publishing Inc. This work is licensed under the Creative Commons Attribution International License (CC BY 4.0).

http://creativecommons.org/licenses/by/4.0/

\section{(c) (i) Open Access}

\begin{abstract}
By means of expansions of rapidly in infinity decreasing functions in delta functions and their derivatives, we derive generalized boundary conditions of the Sturm-Liouville equation for transitions and barriers or wells between two asymptotic potentials for which the solutions are supposed as known. We call such expansions "moment series" because the coefficients are determined by moments of the function. An infinite system of boundary conditions is obtained and it is shown how by truncation it can be reduced to approximations of a different order (explicitly made up to third order). Reflection and refraction problems are considered with such approximations and also discrete bound states possible in nonsymmetric and symmetric potential wells are dealt with. This is applicable for large wavelengths compared with characteristic lengths of potential changes. In Appendices we represent the corresponding foundations of Generalized functions and apply them to barriers and wells and to transition functions. The Sturm-Liouville equation is not only interesting because some important second-order differential equations can be reduced to it but also because it is easier to demonstrates some details of the derivations for this one-dimensional equation than for the full three-dimensional vectorial equations of electrodynamics of media. The article continues a paper that was made long ago.
\end{abstract}

\section{Keywords}

Schrödinger Equation, Drude Approximation, Transition Layer, Potential Barrier, Potential Well, Reflection, Refraction, Moment Series, Generalized Functions, Delta Function and Its Derivatives, Discrete or Bound Eigenstates

\section{Introduction}

It is well-known that differential equations in physics with discontinuous ma- 
terial properties can be solved by applying boundary conditions on the interface between the different media for the adaption of the primary independent solutions for the media. When the transitions between the different media are sufficiently abrupt, but still smooth, one can often solve such problems approximately by generalized boundary conditions. We will demonstrate this here for one of the simplest second-order differential equations in physics, the Sturm-Liouville equation. This equation is of great importance because it is almost identical to the one-dimensional Schrödinger equation and some special problems of wave propagation in optics can be also reduced to this equation.

Generalized boundary conditions in optics taking into account transition layers between two different media were first derived by Drude [1] [2]. Three additional parameters (of the kind of moments) $l=\int_{1}^{2} \mathrm{~d} z, \quad p=\int_{1}^{2} \mathrm{~d} z \varepsilon, \quad q=\int_{1}^{2} \frac{\mathrm{d} z}{\varepsilon} \quad(\varepsilon$ permittivity) play a role, where the integration goes over the boundaries of "thin" transition layers with thickness $I$ which, however, is difficult to define exactly. In reflection and refraction formulae they often go at once into numerator and denominator and partially compensate the incorrectness connected with this approach. They were applied, for example, in the monographs about thin films of Mayer [3], Heavens [4], Rozenberg [5], and Vašiček [6].

A relatively new procedure to derive generalized boundary conditions is to involve generalized functions and, in particular, the step function and all its derivatives which are the delta function with its derivatives. This method is appropriate in classical electrodynamics for the derivation of generalized boundary conditions for media with spatial dispersion [7] (including, e.g., optical activity) and for correct derivations in presence of transition layers. A preliminary step to such derivations of boundary conditions was made in [8] [9]. The method was also developed in [10] [11] in application to the optics of media with transition layers, and the reflection was calculated in the first-order approximation of the generalized boundary conditions. In our paper [12] (see also Acknowledgement) we developed this method for light propagation in media with spatial dispersion (e.g., optical activity or gyrotropy) and transition layers and tried to represent the general procedure of continuing it to higher-order approximations. The main role played expansions of rapidly in infinity decreasing functions into series of delta functions and their derivatives which we call moment series because their coefficients are moments of the expanded function. The further development in the field of reflection and refraction not considered here can be seen, in particular, in the monograph of Lekner [13]. It is fairly comprehensive and represents many different aspects of reflection and refraction in optics and acoustics. In chap. 3 it uses integral invariants which are partially analogous to the parameters which we call moments of a function and in chap. 18 it considers chiral media including boundary conditions (a special case of spatial dispersion [7] which in the new edition of vol. 8 of Landau and Lifshits [14], chap. XII and also in our paper [12] appear under this last name with one of its special cases as natural optical activity). 
In the present article we try to demonstrate the method of derivation of generalized boundary conditions in detail at a simple one-dimensional equation and as such we have chosen the Sturm-Liouville equation (e.g., special monographs [15] [16] [17] and many books of functional analysis) which is nearly the one-dimensional Schrödinger equation and to which many other equations can be reduced (e.g., article [18] in [19]). We need for this purpose exact solutions for asymptotic potentials of the one-dimensional Schrödinger equation (e.g., [20] [21] [22] [23] and others). As said it is easier to make and to explain for this equation the derivations of generalized boundary conditions between two asymptotic potentials up to high-order approximations than for the full Maxwell equations with transition layers between two media and with spatial dispersion in the constitutive equations.

In Section 2 we discuss the restrictions for the applicability of the approach to potential problems for the one-dimensional Schrödinger equation. From Section 3 on we develop systematically the approach of the derivation of boundary conditions for the Sturm-Liouville equation between two asymptotic potentials with solutions supposed as known, in principle. In Section 4 we show how we can obtain finite approximations of the infinite system of boundary conditions and explain the special new situation when we go to the third-order approximation. From Section 5 on we apply this to the special case of two different but asymptotically constant potentials and calculate reflection and refraction problems in Section 6 and discuss in Sections 7 and 8 the appearance of discrete eigenstates which are in certain sense equivalent to total reflection and to surface waves for the three-dimensional Maxwell equations. In Section 9 we give a matrix form of the boundary condition which is well appropriate for a layered system where the output of one layer may serve as input of the next layer. The Appendix A gives a detailed derivation of the moment series in delta functions and their derivatives with moments of the expanded functions as coefficients and some related formulae for Generalized functions. Appendix B brings special examples for such expansions which are interesting for potential barriers and wells and for transition between potentials.

\section{One-Dimensional Schrödinger Equation for Potential Functions and the de-Broglie Wavelength}

The one-dimensional time-dependent Schrödinger equation for a particle with mass $m$ in a state $|\Psi(t)\rangle$ in abstract representation possesses the form

$$
\mathrm{i} \hbar \frac{\partial}{\partial t}|\Psi(t)\rangle=H|\Psi(t)\rangle, \quad(H \equiv T(P)+U(Q)),
$$

where $H$ is the Hamilton operator which is the sum of the operator of kinetic energy $T(P)$

$$
T(P)=\frac{P^{2}}{2 m},
$$

and of potential energy $U(Q)$. With $P$ and $Q$ we denote a pair of canonical 
coordinates which in quantum mechanics obey the commutation relations

$$
[Q, P] \equiv Q P-P Q=\mathrm{i} \hbar I,
$$

where $I$ is the identity operator in representation (Hilbert) space. In representation of the (non-normalizable) eigenstates $|q\rangle$ of the operator $Q$ according to

$$
\begin{aligned}
& Q|q\rangle=q|q\rangle, \quad\left\langle q\left|Q=q\langle q|, \quad P| q\rangle=\mathrm{i} \hbar \frac{\partial}{\partial q}\right| q\right\rangle, \\
& \langle q| P=-\mathrm{i} \hbar \frac{\partial}{\partial q}\langle q|, \quad\left\langle q^{\prime} \mid q\right\rangle=\delta\left(q-q^{\prime}\right),
\end{aligned}
$$

we find from (2.1) the time-dependent Schrödinger equation in $q$-representation ${ }^{1}$

$$
\mathrm{i} \hbar \frac{\partial}{\partial t} \Psi(q, t)=\left\{-\frac{\hbar^{2}}{2 m} \frac{\partial^{2}}{\partial q^{2}}+U(q)\right\} \Psi(q, t), \quad \Psi(q, t) \equiv\langle q \mid \Psi(t)\rangle .
$$

With the supposition

$$
\Psi(q, t)=\exp \left(-\mathrm{i} \frac{E t}{\hbar}\right) \psi_{0}(q, E),
$$

where $E$ is the energy of the particle one obtains from (2.1) the time-independent Schrödinger equation

$$
\left\{-\frac{\hbar^{2}}{2 m} \frac{\partial^{2}}{\partial q^{2}}+U(q)\right\} \psi_{0}(q, E)=E \psi_{0}(q, E) .
$$

It will be taken as our starting equation for the derivation of boundary conditions for the approximate solution of (one-dimensional) potential problems.

First, we make an estimation of the order of magnitudes with which we have to do in calculations with the Schrödinger Equation (2.7) if $q$ is a spatial coordinate. Starting from Einstein's equivalence of mass $m$ and energy $E=m c^{2}$ de Broglie [24] conjectured that particles due to the dualism of waves and particles should possess also a wavelength which in analogy to Planck's and Einstein's relations for the energy $E$ of particles and light is given by the momentum $\boldsymbol{p}$ together with the dispersion equation and that according to hypothesis it is determined by (clight velocity, $m_{0}$ rest mass, $E_{0}$ rest energy)

$$
E=\hbar \omega, \quad \boldsymbol{p}=\hbar \boldsymbol{k}, \quad E^{2}-c^{2} \boldsymbol{p}^{2}=\left(m_{0} c^{2}\right)^{2}, \quad E_{0}=m_{0} c^{2} .
$$

Herein $\omega$ is the (circular) frequency, $\boldsymbol{k}$ the wave vector and $\hbar$ Planck's action quantum (divided by $2 \pi)^{2}$. From classical theory of a free particle it is known and from relativistic theory for small velocities $\frac{\boldsymbol{v}^{2}}{c^{2}}=\frac{c^{2} \boldsymbol{p}^{2}}{E_{0}^{2}} \leq 1$ (or

${ }^{1}$ Due to non-commutativity of the operators $(Q, P)$ a Hamilton operator $H$ by writing it $H(Q, P)$ is not uniquely defined. Fortunately in history of quantum mechanics was that in its simplest form $H=T+U$ two parts which depend on $P$ and $Q$ alone were additively separated and the ordering problem of these operators was not stringent.

${ }^{2}$ According to the practice of that time de Broglie used as notations for Planck's action quantum $h \equiv 2 \pi \hbar$ and instead of the circular frequency $\omega$ the usual frequency $v \equiv \frac{\omega}{2 \pi}$ that means $E=h v=\hbar \omega$ 
$\left.E \approx E_{0}\right)$ it follows that its kinetic energy is

$$
E_{\text {kin }} \equiv E-E_{0} \approx \frac{\boldsymbol{p}^{2}}{2 m_{0}}=\frac{\hbar^{2} \boldsymbol{k}^{2}}{2 m_{0}},
$$

and from this dispersion equation resolved to $|\boldsymbol{p}|=\hbar|\boldsymbol{k}|=\hbar \frac{2 \pi}{\lambda}=\sqrt{2 m_{0} E_{\mathrm{kin}}}$ one obtains for the wavelength $\lambda$ of the moving free particles with velocity $v \equiv|v|$ and momentum $\boldsymbol{p}=m_{0} \boldsymbol{v}$ and for frequency $v$

$$
\lambda \approx \frac{2 \pi \hbar}{\sqrt{2 m_{0} E_{\mathrm{kin}}}}=\left(\frac{h}{m_{0} c}\right) \sqrt{\frac{m_{0} c^{2}}{2 E_{\mathrm{kin}}}}=\frac{h}{m_{0} v}, \quad v \approx \frac{m_{0} c^{2}}{2 \pi \hbar} .
$$

From this dispersion relations follows a phase velocity $\frac{\omega}{|\boldsymbol{k}|}$ larger than $c$ (is not a signal velocity)

$$
\frac{\omega}{|\boldsymbol{k}|} \equiv \lambda v \approx c \sqrt{\frac{E_{0}}{2 E_{\mathrm{kin}}}} \gg c
$$

and from (2.8) the group velocity $\boldsymbol{v} \equiv \frac{\partial \omega}{\partial \boldsymbol{k}}$ for the free particle waves equal to particle velocity $\boldsymbol{v}$

$$
\frac{\partial \omega}{\partial \boldsymbol{k}}=\frac{\partial E}{\partial \boldsymbol{p}}=\frac{c^{2} \boldsymbol{p}}{E}=c \frac{c \boldsymbol{k}}{\omega} \approx c\left(\frac{h}{m_{0} c}\right) \frac{\boldsymbol{k}}{2 \pi}=c \frac{v}{c} \frac{\boldsymbol{k}}{|\boldsymbol{k}|} \ll c \frac{\boldsymbol{k}}{|\boldsymbol{k}|} .
$$

The quantity $\frac{h}{m_{0} c}$ is the Compton length of the particle and $m_{0} c^{2}$ the rest energy of the particle. For an electron with mass $m_{\mathrm{e}}$ the following values are well known from experiment

$$
\frac{h}{m_{\mathrm{e}} c} \approx 2.43 \times 10^{-10} \mathrm{~cm}, \quad m_{\mathrm{e}} c^{2} \approx 0.511 \mathrm{MeV}=0.511 \times 10^{6} \mathrm{eV} .
$$

Therefore, the de-Broglie wavelength for a free electron with kinetic energy $E_{\text {kin }}$ is approximately

$$
\lambda_{\mathrm{e}}=\frac{1}{\sqrt{2}}\left(\frac{h}{m_{\mathrm{e}} c}\right) \sqrt{\frac{m_{\mathrm{e}} c^{2}}{E_{\mathrm{kin}}}} \approx 1.23 \times 10^{-7} \sqrt{\frac{1 \mathrm{eV}}{E_{\text {kin }}}} \mathrm{cm} .
$$

For comparison, the first Bohr radius is $a_{0}=\frac{\hbar^{2}}{m_{\mathrm{e}} e^{2}} \approx 5.29 \times 10^{-9} \mathrm{~cm}$ (e elementary charge), and the Rydberg constant $R_{\mathrm{e}}$ (ionization potential) for infinitely high mass of the proton is $R_{\mathrm{e}}=\frac{1}{2} \alpha^{2} m_{\mathrm{e}} c^{2} \approx 13.6 \mathrm{eV} ;(\alpha \approx 1 / 137)$. For energies of the electron up to the order of a few $\mathrm{eV}$ the wavelengths are large in comparison to the first Bohr radius. Furthermore, $1 \mathrm{eV}$ is equivalent to energy $\kappa_{\mathrm{B}} T$ corresponding to temperature $T$ of $11,606 \mathrm{~K}\left(\kappa_{\mathrm{B}}\right.$ Boltzmann constant).

The approximate boundary conditions which we derive in the following for the Schrödinger Equation (2.7) are applicable if the wavelengths $\lambda_{\mathrm{e}}$ (more generally, $\lambda$ ) are large in comparison to the order of lengths where the potential 
function $U(q)$ changes essentially. Oppositely, if the wave length of the particle is small in comparison to the order of lengths where the changes of the potential function play a role then another well-known approximation, the WKB-method ${ }^{3}$ or quasi-classical approximation, can be applied (e.g., [20]). For high kinetic energies $E_{\text {kin }}$ of the order of the rest energy of the electron of about $0.5 \mathrm{MeV}$ and higher the (non-relativistic) Schrödinger equation is no more relevant.

We usually consider in this paper Equation (2.7) in an infinite interval. If $U(q)$ in (2.7) is larger than $U( \pm \infty)$ then one speaks about a potential barrier and if it is smaller than $U( \pm \infty)$ then about a potential well. Both and more general cases which are not to classify in such simple way are included into the following calculations.

With the substitutions which preserve the coordinate as a quantity with dimension of a length

$$
q \equiv z, \quad U(q) \equiv \frac{\hbar^{2}}{2 m} u(z), \quad E \equiv \frac{\hbar^{2}}{2 m} \lambda, \quad \psi_{0}(q, E) \equiv \psi(z, \lambda),
$$

Equation (2.7) can be transformed to the more neutrally looking form ( $\lambda$ now spectral parameter)

$$
\left\{-\frac{\partial^{2}}{\partial z^{2}}+u(z)-\lambda\right\} \psi(z, \lambda)=0 .
$$

This is the standard form of the Sturm-Liouville equation to which also some other differential equations of physics can be reduced as mentioned in the Introduction. An important example is the wave propagation in one-dimensional inhomogeneous media which is described by Landau and Lifshits [14] (chap. X, section 88). The case of polarization of the electric field $\boldsymbol{E}\left(z ; \boldsymbol{k}_{\perp}, \omega\right)$ perpendicular to the incidence plane is more directly connected with Equation (2.16) (substitutions: $u(z) \rightarrow-\frac{\omega^{2}}{c^{2}} \varepsilon(z ; \omega) ; \lambda \rightarrow-\boldsymbol{k}_{\perp}^{2}$ ) than the case of polarization in the incidence plane which requires more subtile substitutions. One has to remove then the arising first-order derivatives in the equation [14] [15] [16]. Central-symmetric potentials in two and three dimensions with transition regions can be dealt with as quasi-one-dimensional cases of the Schrödinger equation and can be transformed to a Sturm-Liouville equation with additional terms to the potential function (effective potentials) and possible applications to scattering problems. We cannot deal with this here.

If $\psi_{1}(z, \lambda)$ and $\psi_{2}(z, \lambda)$ are two solutions of the Sturm-Liouville equation to the same potential $u(z)$ and the same parameter $\lambda$ then in known way one derives the conservation theorem

$$
\frac{\partial}{\partial z}\left(\psi_{1}(z, \lambda) \frac{\partial}{\partial z} \psi_{2}(z, \lambda)-\psi_{2}(z, \lambda) \frac{\partial}{\partial z} \psi_{1}(z, \lambda)\right)=0 .
$$

The same is true if the second solution is the complex-conjugate solution of ${ }^{3}$ WKB stands for "Wentzel, Kramers, Brillouin". 
the first solution for real parameter $\lambda$ that means (we add then a factor "I" to get a real-valued conservation quantity)

$$
\mathrm{i} \frac{\partial}{\partial z}\left(\psi(z, \lambda) \frac{\partial}{\partial z} \psi^{*}(z, \lambda)-\psi^{*}(z, \lambda) \frac{\partial}{\partial z} \psi(z, \lambda)\right)=0, \quad\left(\lambda=\lambda^{*}\right) .
$$

Other general properties we find, for example, in [15] [16] [17] [18].

\section{Sturm-Liouville Equation for Transition Potentials between Two Asymptotic Potentials and Its Solution by Boundary Conditions}

We derive in this Section at an example generalized boundary conditions using the theory of Generalized functions (e.g., [25] [26] [27] [28], see also Appendix A). As starting point of the further treatment we take the following ordinary second-order differential Equation (2.16)

$$
(L-\lambda) \psi(z, \lambda)=0, \quad L \equiv-\frac{\partial^{2}}{\partial z^{2}}+u(z),
$$

which in this form is commonly called the Sturm-Liouville equation. The operator $L$ is the Sturm-Liouville operator and $\lambda$ its eigenvalues. The eigensolutions $\psi=\psi(z, \lambda)$ depend on $\lambda$ as parameter.

We assume that the real-valued potential $u(z)$ is defined on the entire real $z$-axis $(-\infty<z<+\infty)$. The complex-valued solutions $\psi(z, \lambda)$ are considered in dependence on the spectral parameter $\lambda$ which can take on arbitrary complex values. As a linear second-order differential equation, the Sturm-Liouville equation possesses two linearly independent solutions for each complex value of the spectral parameter $\lambda$. As mentioned the Sturm-Liouville Equation (3.1) can also be seen as the eigenvalue problem of the Sturm-Liouville operator $L$. Then the real eigenvalues $\lambda$ of the discrete and of the continuous spectrum may be separated from the totality of complex values of the spectral parameter $\lambda$ by appropriate normalization conditions of the solutions. The one-dimensional Schrödinger equation in energy representation is identical with (3.1) after simple linear substitutions of the variables (2.15), where the energy becomes proportional to $\lambda$. It is by no means a disadvantage to assume that $u(z)$ and $\psi(z, \lambda)$ are Generalized functions and that the derivatives in (2.1) are also Generalized functions because this includes or generalizes all classical cases and the point-wise definition of functions is perhaps an unnecessary (and may be unnatural) concept in physics.

For the purpose of the illustration only the asymptotic potentials $u_{\mp}(z)$ for $z \rightarrow \pm \infty$ are chosen as constant potentials $u_{-}(z)=u(-\infty), u_{+}(z)=u(+\infty)$. The first partial figure shows the genuine potential and below this its approximation by a step function. The upper partial figure on the right-hand side shows in hatched way the error which we make by the approximation and below this the difference of potential and its approximation. The derivations show that the parts to the left and to the right of the chosen boundary are, in general, separately to deal with. 
We assume now that the potential $u(z)$ can be approximated for $z \rightarrow-\infty$ by $u_{-}(z)$ and for $z \rightarrow+\infty$ by $u_{+}(z)$ and that the totality of solutions of the following two Sturm-Liouville equations

$$
0=\left\{-\frac{\partial^{2}}{\partial z^{2}}+u_{\mp}(z)-\lambda\right\} \psi_{\mp}(z, \lambda),
$$

is known. The asymptotic potentials $u_{-}(z)$ and $u_{+}(z)$ have to be of such kind that the differences $u(z)-u_{-}(z)$ and $u(z)-u_{+}(z)$ become rapidly decreasing functions for $z \rightarrow+\infty$ or $z \rightarrow-\infty$, respectively. The rapid decrease of functions in infinity means that they decrease in infinity more rapidly than any power function $|z|^{n}$ with arbitrary negative numbers $n$ or, more generally expressed, that they can be defined as linear continuous functionals over the space of smooth and moderately increasing functions, especially, the polynomials. The effective lengths of the transition regions should be small compared with the characteristic lengths of changes in the solutions for the asymptotic potentials $u_{+}(z)$ and $u_{-}(z)$ (e.g., wavelengths). Usually, $u_{+}(z)$ and $u_{-}(z)$ are chosen as such potentials for which we know the totality of solutions in form of elementary or well-known higher transcendental functions as, for instance, for constant potential (Exponential or Trigonometric functions), linear potential (Airy functions) and quadratic potential (Hermite functions or more generally Parabolic-cylinder functions).

An exact boundary between the two regions of $z \rightarrow-\infty$ and $z \rightarrow+\infty$ is not defined and for approximations of the derived boundary conditions a "good" choice of the reference point $Z_{0}$ is necessary. A "good" choice in our case is $z_{0}=0$ and is drawn in Figure 1. The partial Figures illustrate the influence of the reference point $z_{0}$ onto the hatched correction functions.

There are also some other well-known special potentials with known exact solutions of the Sturm-Liouville equation as, for instance, the reflection-less Bargmann potentials with their importance in soliton theory of the Korteweg-de Vries equation (the famous Hyperbolic Secans square as the simplest nontrivial one). The solutions for the most common potentials can be found, e.g., in Vol. III (Quantum mechanics) of Landau and Lifshitz [20] (chap. 3) and furthermore in [21] (chap. 12.3) and in [22]. The derivation of relations between the asymptotic solutions $\psi_{+}(z, \lambda)$ and $\psi_{-}(z, \lambda)$ belongs to reflection and refraction or scattering problems of the Sturm-Liouville equation. By means of the generalized boundary conditions which we will derive now, one can obtain approximate solutions of the direct scattering problem of Equation (3.1).

The almost smooth transition of the potential $u(z)$ from the asymptotic potential $u_{-}(z)$ for $z \rightarrow-\infty$ to the asymptotic potential $u_{+}(z)$ for $z \rightarrow+\infty$ has to be concentrated mainly on the neighborhood of a value $z_{0}$ of the variable $z$ (Figure 1) which is not exactly determined and can be varied in certain way. We have then to choose a suitable point

$$
z=z_{0},
$$

as the boundary or the reference point of the expansions in the proposed method. 
Continuous transition together with approximations (boundary chosen at $z=0$ )

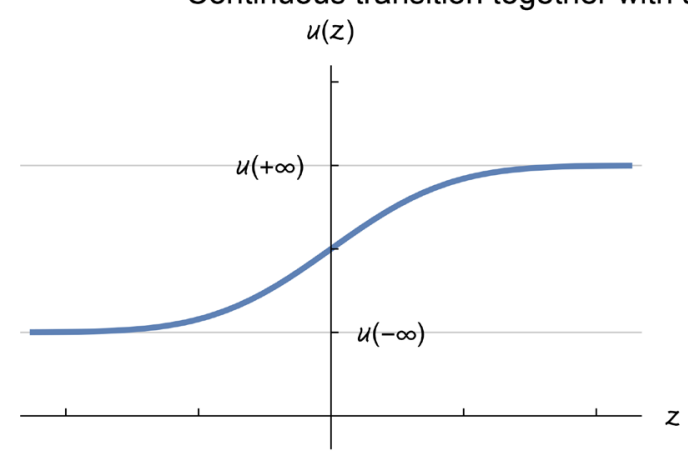

$u(z)$ and $u_{-}(z) \theta(-z)+u_{+}(z) \theta(z)$
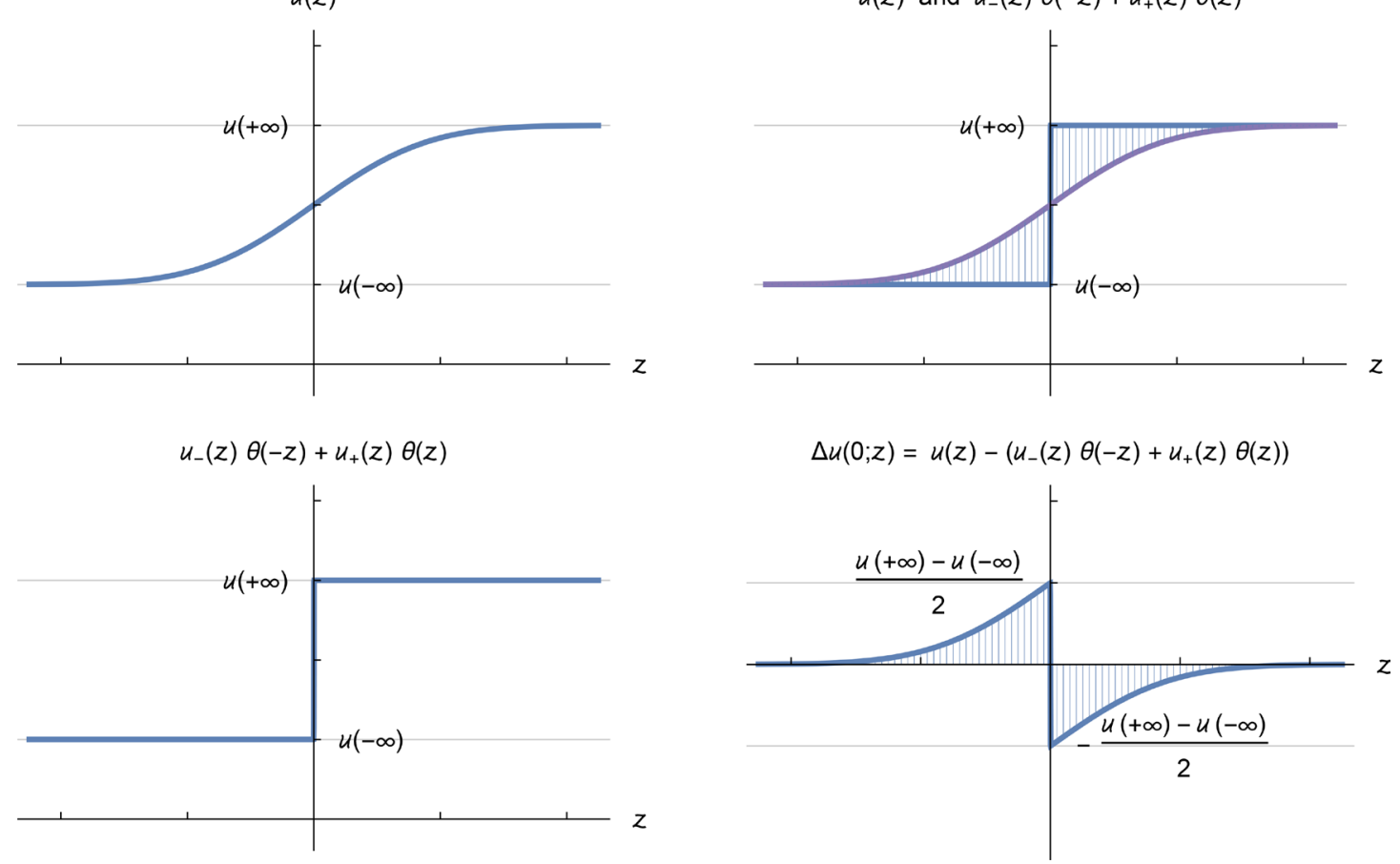

Figure 1. Transition between two constant potentials at minus and plus infinity.
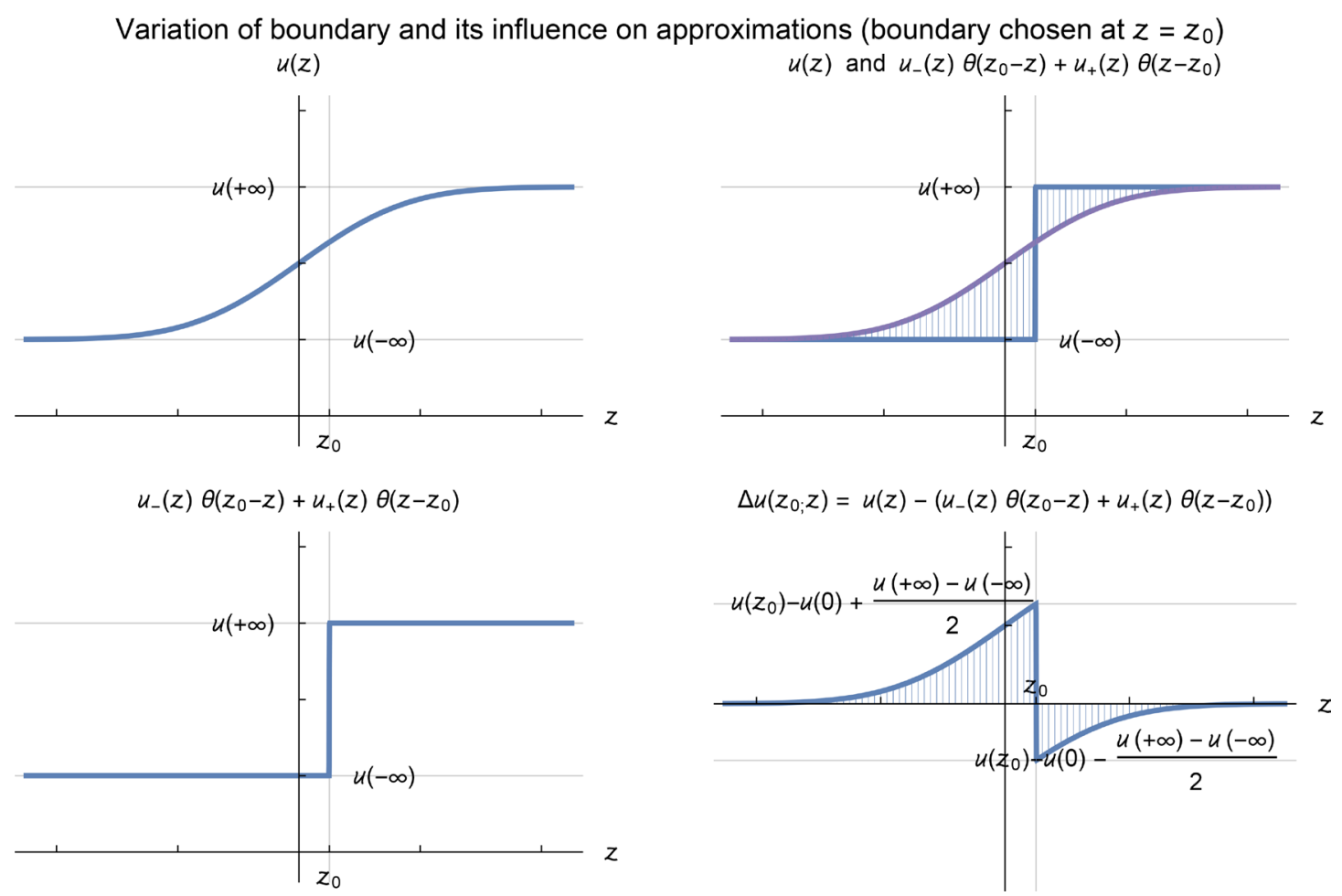

Figure 2. Transition between two constant potentials at infinity with variation of the reference point $z_{0}$ of the boundary.

A small variation of the reference point $z_{0}$ should not destroy the whole approximation (Figure 2). The special case of a potential barrier (Figure 3 ) or a 
potential well (by taking instead negative values of that for the barrier) (Figure 4) we consider more detailed in Sections 6 and 7.

Next we represent the solution $\psi(z, \lambda)$ of Equation (2.16) after separation of Barrier between asymptotic potentials and influence of boundary variation (boundary chosen at $z=z_{0}$ )

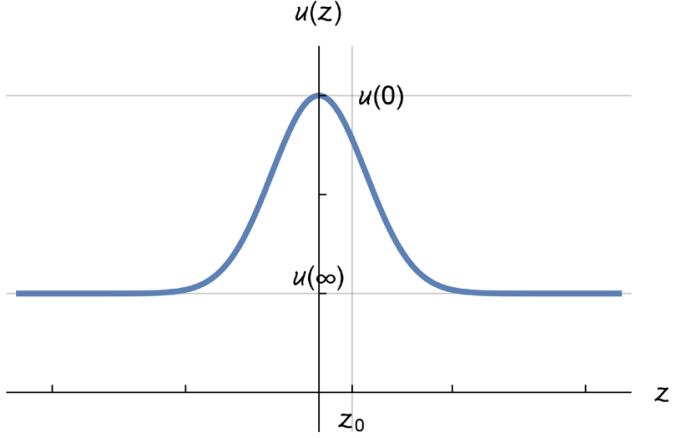

$u(z)=u(\infty)$

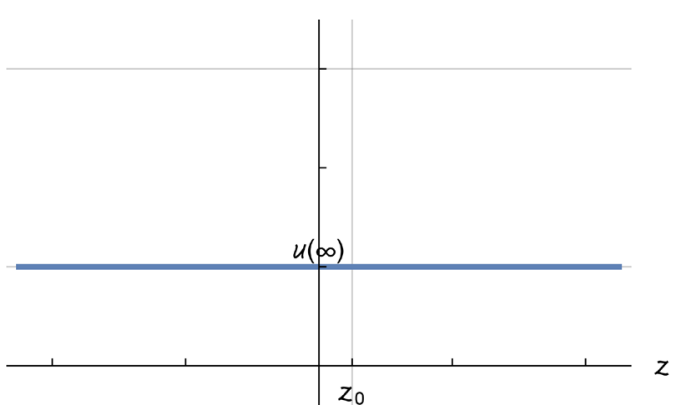

$u(z)$ and $u(z)=u(\infty)$
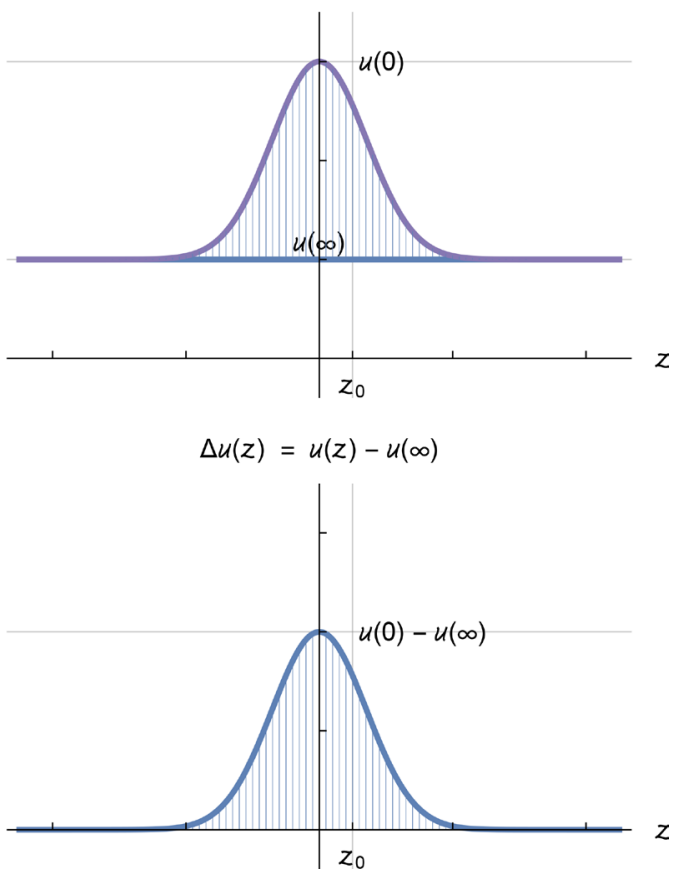

Figure 3. Potential barrier over constant potential at infinity with reference point at $z_{0}$.

Well between asymptotic potentials and influence of boundary variation (boundary chosen at $z=z_{0}$ ) $u(z)$ $u(z)$ and $u(z)=u(\infty)$
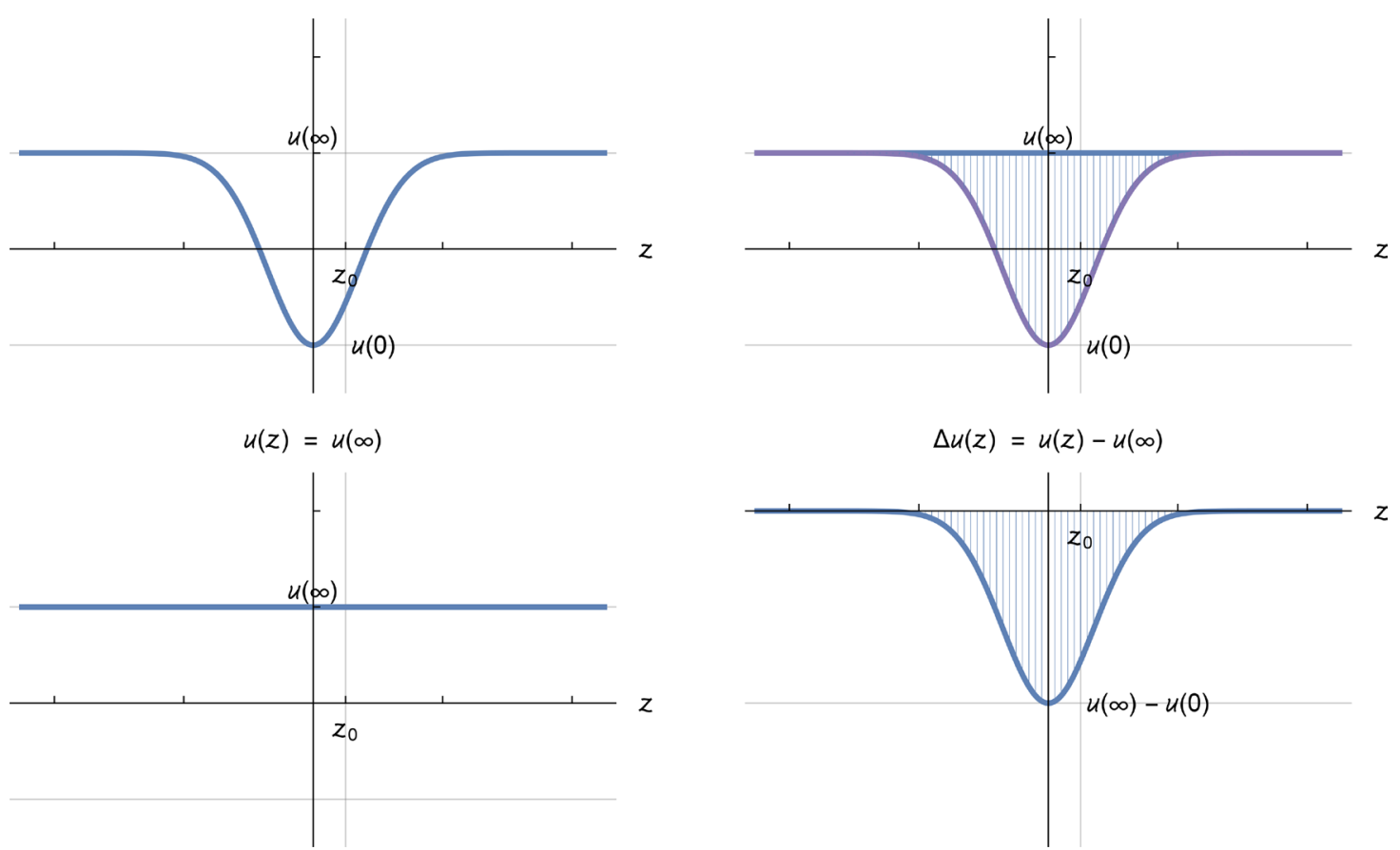

Figure 4. Potential well under constant potential at infinity with reference point at $z_{0}$. 
the asymptotic solutions $\psi_{\mp}(z, \lambda)$ to the asymptotic potentials $u_{\mp}(z)$ by means of Heaviside's step function $\theta(z)$ in the form

$$
\begin{aligned}
(\theta(z)= & 0,(z<0), \theta(z)=1,(z>0)) \\
& \psi(z, \lambda)=\psi_{-}(z, \lambda) \theta\left(z_{0}-z\right)+\psi_{+}(z, \lambda) \theta\left(z-z_{0}\right)+\psi^{\prime}\left(z_{0} ; z, \lambda\right),
\end{aligned}
$$

where $\psi^{\prime}\left(z_{0} ; z, \lambda\right)$ depending in addition on the reference point $z_{0}$ is a small deviation from the sum of the two asymptotic solutions in the transition region and turns out to be a function of $z$ rapidly decreasing for $z \rightarrow \pm \infty$ and mainly concentrated on the neighborhood of the point $Z_{0}$. Below we make a moment-series expansion of $\psi^{\prime}\left(z_{0} ; z, \lambda\right)$ into a delta and its derivatives (see Appendix A).

Inserting (3.4) into the Sturm-Liouville Equation (2.16) the following equation results

$0=\left\{-\frac{\partial^{2}}{\partial z^{2}}+u(z)-\lambda\right\}\left(\psi_{-}(z, \lambda) \theta\left(z_{0}-z\right)+\psi_{+}(z, \lambda) \theta\left(z-z_{0}\right)+\psi^{\prime}\left(z_{0} ; z, \lambda\right)\right)$,

which we have to solve. For this purpose we apply Generalized functions and an expansion of rapidly in infinity decreasing functions into delta function and its derivatives called moment series which we explain in Appendix A.

Using the Leibniz rule for the $n$-fold differentiation of a product of functions $f(z) g(z)$

$$
\frac{\partial^{n}}{\partial z^{n}} f(z) g(z)=\sum_{k=0}^{n} \frac{n !}{(n-k) ! k !} f^{(n-k)}(z) g^{(k)}(z),
$$

we can make the following identical transformation of a part of the right-hand side of (3.5) which brings the Heaviside step functions in front of the derivatives

$$
\begin{aligned}
- & \frac{\partial^{2}}{\partial z^{2}}\left(\psi_{-}(z, \lambda) \theta\left(z_{0}-z\right)+\psi_{+}(z, \lambda) \theta\left(z-z_{0}\right)\right) \\
= & -\theta\left(z_{0}-z\right) \frac{\partial^{2}}{\partial z^{2}} \psi_{-}(z, \lambda)-\theta\left(z-z_{0}\right) \frac{\partial^{2}}{\partial z^{2}} \psi_{+}(z, \lambda) \\
& +2 \delta\left(z-z_{0}\right)\left(\frac{\partial}{\partial z} \psi_{-}(z, \lambda)-\frac{\partial}{\partial z} \psi_{+}(z, \lambda)\right) \\
& +\delta^{(1)}\left(z-z_{0}\right)\left(\psi_{-}(z, \lambda)-\psi_{+}(z, \lambda)\right) \\
= & -\theta\left(z_{0}-z\right) \frac{\partial^{2}}{\partial z^{2}} \psi_{-}(z, \lambda)-\theta\left(z-z_{0}\right) \frac{\partial^{2}}{\partial z^{2}} \psi_{+}(z, \lambda) \\
& +\delta\left(z-z_{0}\right)\left(\psi_{-}^{(1)}\left(z_{0}, \lambda\right)-\psi_{+}^{(1)}\left(z_{0}, \lambda\right)\right) \\
& +\delta^{(1)}\left(z-z_{0}\right)\left(\psi_{-}\left(z_{0}, \lambda\right)-\psi_{+}\left(z_{0}, \lambda\right)\right) .
\end{aligned}
$$

We have applied the identity (A.5) to make the coefficients of the delta function and its first derivative independent on variable $z$. Equation (3.5) using this identity can be written

$$
\begin{aligned}
0= & \theta\left(z_{0}-z\right)\left\{-\frac{\partial^{2}}{\partial z^{2}}+u(z)-\lambda\right\} \psi_{-}(z, \lambda) \\
& +\theta\left(z-z_{0}\right)\left\{-\frac{\partial^{2}}{\partial z^{2}}+u(z)-\lambda\right\} \psi_{+}(z, \lambda)
\end{aligned}
$$




$$
\begin{aligned}
& +\delta\left(z-z_{0}\right)\left(\psi_{-}^{(1)}\left(z_{0}, \lambda\right)-\psi_{+}^{(1)}\left(z_{0}, \lambda\right)\right) \\
& +\delta^{(1)}\left(z-z_{0}\right)\left(\psi_{-}\left(z_{0}, \lambda\right)-\psi_{+}\left(z_{0}, \lambda\right)\right) \\
& +\left\{-\frac{\partial^{2}}{\partial z^{2}}+u(z)-\lambda\right\} \psi^{\prime}\left(z_{0} ; z, \lambda\right) .
\end{aligned}
$$

With the asymptotic Equations (3.2) we may transform this equation to

$$
\begin{aligned}
0= & +\delta\left(z-z_{0}\right)\left(\psi_{-}^{(1)}\left(z_{0}, \lambda\right)-\psi_{+}^{(1)}\left(z_{0}, \lambda\right)\right) \\
& +\delta^{(1)}\left(z-z_{0}\right)\left(\psi_{-}\left(z_{0}, \lambda\right)-\psi_{+}\left(z_{0}, \lambda\right)\right) \\
& +\theta\left(z_{0}-z\right)\left(u(z)-u_{-}(z)\right) \psi_{-}(z, \lambda) \\
& +\theta\left(z-z_{0}\right)\left(u(z)-u_{+}(z)\right) \psi_{+}(z, \lambda) \\
& +\left\{-\frac{\partial^{2}}{\partial z^{2}}+u(z)-\lambda\right\} \psi^{\prime}\left(z_{0} ; z, \lambda\right) .
\end{aligned}
$$

Our aim is to represent this equation as a superposition of the delta function and of its derivatives $\delta^{(n)}\left(z-z_{0}\right),(n=0,1,2, \cdots)$ with respect to variable $z$ where the coefficients should not depend on variable $z$. For the sum term with the function $\psi^{\prime}\left(z_{0} ; z, \lambda\right)$ we make this below and consider now the two sum terms in the second line of (3.9).

The deviation $\Delta u\left(z_{0} ; z\right)$ of the potential $u(z)$ in the transition region from the two asymptotic potentials $u_{-}(z)$ and $u_{+}(z)$ depends on the chosen boundary $z_{0}$ and will be defined by

$$
\begin{aligned}
\Delta u\left(z_{0} ; z\right) & \equiv u(z)-u_{-}(z) \theta\left(z_{0}-z\right)-u_{+}(z) \theta\left(z-z_{0}\right) \\
& =\left(u(z)-u_{-}(z)\right) \theta\left(z_{0}-z\right)+\left(u(z)-u_{+}(z)\right) \theta\left(z-z_{0}\right) .
\end{aligned}
$$

However, since it does not find entrance in Equation (3.9) as a whole but is combined with the solutions $\psi_{-}(z, \lambda)$ and $\psi_{+}(z, \lambda)$ we have to deal with the two parts $u(z)-u_{-}(z)$ for $z \leq z_{0}$ and $u(z)-u_{+}(z)$ for $z \geq z_{0}$ separately.

Since $u(z)-u_{-}(z)$ and $u(z)-u_{+}(z)$ by presupposition are concentrated in the neighborhood of the chosen boundary $z=z_{0}$ and are rapidly vanishing for $z \rightarrow \mp \infty$ one may make moment-series expansions of the form (see Equations (A.23) and (A.24) in Appendix A)

$$
\begin{aligned}
& \left(u(z)-u_{-}(z)\right) \theta\left(z_{0}-z\right)=\sum_{m=0}^{\infty} u_{-, m}\left(z_{0}\right) \delta^{(m)}\left(z-z_{0}\right), \\
& \left(u(z)-u_{+}(z)\right) \theta\left(z-z_{0}\right)=\sum_{m=0}^{\infty} u_{+, m}\left(z_{0}\right) \delta^{(m)}\left(z-z_{0}\right),
\end{aligned}
$$

with coefficients $u_{-, m}\left(z_{0}\right)$ and $u_{+, m}\left(z_{0}\right)$ which are left-hand and right-hand moments of the hatched error parts of the potential which do not depend on variable $z$ (but on reference point $z_{0}$ ) and are given by

$$
\begin{aligned}
u_{-, m}\left(z_{0}\right) & \equiv \frac{(-1)^{m}}{m !} \int_{-\infty}^{z_{0}} \mathrm{~d} z\left(u(z)-u_{-}(z)\right)\left(z-z_{0}\right)^{m} \\
& =\frac{1}{m !} \int_{-\infty}^{z_{0}} \mathrm{~d} z\left(u(z)-u_{-}(z)\right) \underbrace{\left(z_{0}-z\right)^{m}}_{\geq 0},
\end{aligned}
$$




$$
u_{+, m}\left(z_{0}\right) \equiv \frac{(-1)^{m}}{m !} \int_{z_{0}}^{+\infty} \mathrm{d} z\left(u(z)-u_{+}(z)\right) \underbrace{\left(z-z_{0}\right)^{m}}_{\geq 0} .
$$

These are integrals over the hatched parts in last partial figure in Figure 2 multiplied by $\left(z-z_{0}\right)^{m}$.

We have now to multiply the expressions in (3.11) with $\psi_{-}(z, \lambda)$ and $\psi_{+}(z, \lambda)$, respectively, and using the multiplication formula (A.5) after reordering of the arising double sums we obtain

$$
\begin{aligned}
& \left(u(z)-u_{-}(z)\right) \theta\left(z_{0}-z\right) \psi_{-}(z, \lambda)+\left(u(z)-u_{+}(z)\right) \theta\left(z-z_{0}\right) \psi_{+}(z, \lambda) \\
& =\sum_{m=0}^{\infty}\left(u_{-, m}\left(z_{0}\right) \delta^{(m)}\left(z-z_{0}\right) \psi_{-}(z, \lambda)+u_{+, m}\left(z_{0}\right) \delta^{(m)}\left(z-z_{0}\right) \psi_{+}(z, \lambda)\right) \\
& =\sum_{m=0}^{\infty} \sum_{k=0}^{m} \frac{(-1)^{k} m !}{k !(m-k) !}\left(u_{-, m}\left(z_{0}, \lambda\right) \psi_{-}^{(k)}\left(z_{0}, \lambda\right)+u_{+, m}\left(z_{0}, \lambda\right) \psi_{+}^{(k)}\left(z_{0}, \lambda\right)\right) \delta^{(m-k)}\left(z-z_{0}\right) \\
& =\sum_{n=0}^{\infty} \sum_{k=0}^{\infty} \frac{(-1)^{k}(n+k) !}{n ! k !}\left(u_{-, n+k}\left(z_{0}\right) \psi_{-}^{(k)}\left(z_{0}, \lambda\right)+u_{+, n+k}\left(z_{0}\right) \psi_{+}^{(k)}\left(z_{0}, \lambda\right)\right) \delta^{(n)}\left(z-z_{0}\right),
\end{aligned}
$$

The hatched error function to the approximation does not depend on the reference point $z_{0}$ but the moments depend on it. However, the first moment $\int_{-\infty}^{+\infty} \mathrm{d} z(u(z)-u(\infty))$ is independent of the chosen reference point $z_{0}$. Similar is the case of a potential well between two asymptotic equal potentials by changing the sign of $u(z)$.

The hatched error function to the approximation does not depend on the reference point $z_{0}$ but the moments depend on it. However, the first moment $\int_{-\infty}^{+\infty} \mathrm{d} z(u(z)-u(\infty))$ is independent of the chosen reference point $z_{0}$.

There remains to represent the additional term $\psi^{\prime}\left(z_{0} ; z, \lambda\right)$ to the asymptotic solution in (3.5) which is concentrated onto the neighborhood of $z=z_{0}$ as a superposition of the delta function and of their derivatives that means the expansion into a moment series according to (see Appendix A)

$$
\psi^{\prime}\left(z_{0} ; z, \lambda\right)=\sum_{m=0}^{\infty} \psi_{m}^{\prime}\left(z_{0}, \lambda\right) \delta^{(m)}\left(z-z_{0}\right),
$$

The hatched error function to the approximation does not depend on the reference point $z_{0}$ but the moments dependon it. However, the first moment $\int_{-\infty}^{+\infty} \mathrm{d} z(u(z)-u(\infty))$ is independent of the chosen reference point $z_{0}$.

With the coefficients $\psi_{m}^{\prime}\left(z_{0}, \lambda\right)$ which do not depend on variable $z$ but on the reference point $z_{0}$

$$
\psi_{m}^{\prime}\left(z_{0}, \lambda\right) \equiv \frac{(-1)^{m}}{m !} \int_{-\infty}^{+\infty} \mathrm{d} z \psi^{\prime}\left(z_{0} ; z, \lambda\right)\left(z-z_{0}\right)^{m} .
$$

Now we have to apply the operator $L-\lambda$ of the Sturm-Liouville equation to the moment-series expansion of the "small" additional part $\psi^{\prime}\left(z_{0} ; z, \lambda\right)$ of the solution. Using the multiplication theorem (A.5) and the expansion (3.14) we find

$$
-\frac{\partial^{2}}{\partial z^{2}} \psi^{\prime}\left(z_{0} ; z, \lambda\right)=-\sum_{n=2}^{\infty} \psi_{n-2}^{\prime}\left(z_{0}, \lambda\right) \delta^{(n)}\left(z-z_{0}\right),
$$




$$
\begin{gathered}
u(z) \psi^{\prime}\left(z_{0} ; z, \lambda\right)=\sum_{n=0}^{\infty}\left(\sum_{k=0}^{\infty}(-1)^{k} \frac{(n+k) !}{n ! k !} u^{(k)}\left(z_{0}\right) \psi_{n+k}^{\prime}\left(z_{0}, \lambda\right)\right) \delta^{(n)}\left(z-z_{0}\right) \\
-\lambda \psi^{\prime}\left(z_{0} ; z, \lambda\right)=-\lambda \sum_{n=0}^{\infty} \psi_{n}^{\prime}\left(z_{0}, \lambda\right) \delta^{(n)}\left(z-z_{0}\right) .
\end{gathered}
$$

Thus we have prepared the representation of all parts for insertion into Equation (3.9).

Inserting now (3.13) and (3.16) into Equation (3.9) and collecting all terms proportional to $\delta\left(z-z_{0}\right)$ and to its derivatives $\delta^{(n)}\left(z-z_{0}\right),(n=1,2, \cdots)$ we find

$$
\begin{aligned}
0= & \left\{\psi_{-}^{(1)}\left(z_{0}, \lambda\right)-\psi_{+}^{(1)}\left(z_{0}, \lambda\right)+\sum_{k=0}^{\infty}(-1)^{k}\left(u_{-, k}\left(z_{0}\right) \psi_{-}^{(k)}\left(z_{0}, \lambda\right)\right.\right. \\
& \left.\left.+u_{+, k}\left(z_{0}\right) \psi_{+}^{(k)}\left(z_{0}, \lambda\right)+u^{(k)}\left(z_{0}\right) \psi_{k}^{\prime}\left(z_{0}, \lambda\right)\right)-\lambda \psi_{0}^{\prime}\left(z_{0}, \lambda\right)\right\} \delta\left(z-z_{0}\right) \\
& +\left\{\psi_{-}\left(z_{0}, \lambda\right)-\psi_{+}\left(z_{0}, \lambda\right)+\sum_{k=0}^{\infty}(-1)^{k} \frac{(1+k) !}{1 ! k !}\left(u_{-, 1+k}\left(z_{0}\right) \psi_{-}^{(k)}\left(z_{0}, \lambda\right)\right.\right. \\
& \left.\left.+u_{+, 1+k}\left(z_{0}\right) \psi_{+}^{(k)}\left(z_{0}, \lambda\right)+u^{(k)}\left(z_{0}, \lambda\right) \psi_{1+k}^{\prime}\left(z_{0}, \lambda\right)\right)-\lambda \psi_{1}^{\prime}\left(z_{0}, \lambda\right)\right\} \delta^{(1)}\left(z-z_{0}\right) \\
& +\sum_{n=2}^{\infty}\left\{\sum _ { k = 0 } ^ { \infty } ( - 1 ) ^ { k } \frac { ( n + k ) ! } { n ! k ! } \left(u_{-, n+k}\left(z_{0}\right) \psi_{-}^{(k)}\left(z_{0}, \lambda\right)+u_{+, n+k}\left(z_{0}\right) \psi_{+}^{(k)}\left(z_{0}, \lambda\right)\right.\right. \\
& \left.\left.+u^{(k)}\left(z_{0}\right) \psi_{n+k}^{\prime}\left(z_{0}, \lambda\right)\right)-\left(\psi_{n-2}^{\prime}\left(z_{0}, \lambda\right)+\lambda \psi_{n}^{\prime}\left(z_{0}, \lambda\right)\right)\right\} \delta^{(n)}\left(z-z_{0}\right) .
\end{aligned}
$$

All coefficients in front of the derivatives of the delta functions in this equation are now independent on the variable $z$ and according to (3.12) and (3.13) they have to vanish since the left-hand side of (3.17) vanishes. This leads to the intended boundary conditions.

Summarizing, the whole solution of the Sturm-Liouville equation was taken in the general form

$\psi(z, \lambda)=\psi_{-}(z, \lambda) \theta\left(z_{0}-z\right)+\psi_{+}(z, \lambda) \theta\left(z-z_{0}\right)+\sum_{n=0}^{\infty} \psi_{n}^{\prime}\left(z_{0}, \lambda\right) \delta^{(n)}\left(z-z_{0}\right)$,

and after application of the Sturm-Liouville operator to (3.18) it has to vanish and by separation of the asymptotic solutions (3.2) there remains a part which could be represented as a superposition of the delta function and its derivatives with constant coefficients (3.17) which do not depend on the variable $z$ but only on the chosen boundary at $z=z_{0}$ and the parameter $\lambda$ and all the coefficients have to vanish. In this way we arrived at the intended boundary conditions. Since this system of boundary conditions is infinite we have to look for approximations. This is done in next Section.

\section{The Infinite System of Boundary Conditions and Their Lowest Three Approximations}

The boundary conditions which are as explained the vanishing of the ( $z$-independent) coefficients in front of the delta function and of its derivatives in (3.17) 
are the following:

1) Vanishing of coefficient in front of $\delta\left(z-z_{0}\right)$

$$
\begin{aligned}
0= & \psi_{-}^{(1)}\left(z_{0}, \lambda\right)-\psi_{+}^{(1)}\left(z_{0}, \lambda\right)+\sum_{k=0}^{\infty}(-1)^{k}\left(u_{-, k}\left(z_{0}\right) \psi_{-}^{(k)}\left(z_{0}, \lambda\right)\right. \\
& \left.+u_{+, k}\left(z_{0}\right) \psi_{+}^{(k)}\left(z_{0}, \lambda\right)+u^{(k)}\left(z_{0}\right) \psi_{k}^{\prime}\left(z_{0}, \lambda\right)\right)-\lambda \psi_{0}^{\prime}\left(z_{0}, \lambda\right),
\end{aligned}
$$

2) Vanishing of coefficient in front of $\delta^{(1)}\left(z-z_{0}\right)$

$$
\begin{aligned}
0= & \psi_{-}\left(z_{0}, \lambda\right)-\psi_{+}\left(z_{0}, \lambda\right)+\sum_{k=0}^{\infty}(-1)^{k}(1+k)\left(u_{-, 1+k}\left(z_{0}\right) \psi_{-}^{(k)}\left(z_{0}, \lambda\right)\right. \\
& \left.+u_{+, 1+k}\left(z_{0}\right) \psi_{+}^{(k)}\left(z_{0}, \lambda\right)+u^{(k)}\left(z_{0}\right) \psi_{1+k}^{\prime}\left(z_{0}, \lambda\right)\right)-\lambda \psi_{1}^{\prime}\left(z_{0}, \lambda\right),
\end{aligned}
$$

3) Vanishing of coefficients in front of $\delta^{(n)}\left(z-z_{0}\right),(n=2,3,4, \cdots)$

$$
\begin{aligned}
0= & \sum_{k=0}^{\infty}(-1)^{k} \frac{(n+k) !}{n ! k !}\left(u_{-, n+k}\left(z_{0}\right) \psi_{-}^{(k)}\left(z_{0}, \lambda\right)+u_{+, n+k}\left(z_{0}\right) \psi_{+}^{(k)}\left(z_{0}, \lambda\right)\right. \\
& \left.+u^{(k)}\left(z_{0}\right) \psi_{n+k}^{\prime}\left(z_{0}, \lambda\right)\right)-\left(\psi_{n-2}^{\prime}\left(z_{0}, \lambda\right)+\lambda \psi_{n}^{\prime}\left(z_{0}, \lambda\right)\right) .
\end{aligned}
$$

Apart from the given asymptotic potentials $u_{\mp}(z)$ the properties of the complete potential function $u(z)$ are contained in the moments $u_{\mp, m}\left(z_{0}\right)$ (lower indices $m=0,1,2, \cdots)$ defined in (3.12) and in the $k$-th derivatives $u^{(k)}\left(z_{0}\right)$ of $u(z)$ at reference point $z=z_{0}$ (upper indices $(k=0,1,2, \cdots)$ ) and their definitions are given in last Section, where functions with lower indices mean moments and with upper indices derivatives of the corresponding functions.

All approximations of the boundary condition should be contained in (4.1), (4.2) and (4.3) but truncations for approximations have to be made in non-contradictory way. A difficulty is that in each of the conditions an infinite number of potential properties (summations over $k$ ) is involved and therefore that the truncation is not a simple problem. The properties of the transition region between the two asymptotic parts is described after the choice of a reference point $z_{0}$ by the moments $u_{\mp, n}\left(z_{0}\right)$ defined by (3.12) and in connection with (3.11). As $N$-th approximation we will denote the case where all moments $n \geq N$ are neglected that means

$$
u_{\mp, n}\left(z_{0}\right)=0, \quad(n \geq N) .
$$

It seems that an appropriate strategy to obtain finite approximation of boundary condition is to truncate the expansion of the solution (3.18) from a finite $n \geq N$ on as follows

$$
\psi_{n}\left(z_{0}, \lambda\right)=0, \quad(n \geq N) .
$$

Then the approximate solution possesses the form

$\psi(z, \lambda)=\psi_{-}(z, \lambda) \theta\left(z_{0}-z\right)+\psi_{+}(z, \lambda) \theta\left(z-z_{0}\right)+\sum_{n=0}^{N-1} \psi_{n}^{\prime}\left(z_{0}, \lambda\right) \delta^{(n)}\left(z-z_{0}\right)$.

We call both conditions (4.4) and (4.5) taken together the $N$-th approximation of the boundary conditions. The boundary conditions (4.3) are then identically 
satisfied in $N$-th approximation for $n \geq N+2$ and have to be not taken into account for $n \geq N$. Thus we have $N+2$ boundary conditions for $N+4$ quantities $\psi_{\mp}^{(1)}\left(z_{0}, \lambda\right)$ and $\psi_{\mp}\left(z_{0}, \lambda\right)$ (4 quantities) plus $\psi_{0}^{\prime}\left(z_{0}, \lambda\right), \cdots, \psi_{N-1}^{\prime}\left(z_{0}, \lambda\right)$ ( $N$ quantities) in the $N$-th approximation. For $N \rightarrow \infty$ all terms in the boundary conditions are taken into account. In a sufficiently high approximation one may neglect additional terms if they prove to be negligible. We now consider explicitly the three lowest approximations to the basic boundary conditions (or zeroth approximation).

\subsection{Zeroth-Order or Basic Approximation of the Boundary Conditions}

In the zeroth-order (or basic) approximation we consider the solution in the approximate form

$$
\psi(z, \lambda)=\psi_{-}(z, \lambda) \theta\left(z_{0}-z\right)+\psi_{+}(z, \lambda) \theta\left(z-z_{0}\right)
$$

and neglect all moments, i.e. $u_{\mp, n}\left(z_{0}\right)=0,(n \geq 0)$ for the transition region. This leads according to (4.1) and (4.2) to the basic boundary conditions

$$
\begin{gathered}
0=\psi_{-}^{(1)}\left(z_{0}, \lambda\right)-\psi_{+}^{(1)}\left(z_{0}, \lambda\right), \\
0=\psi_{-}\left(z_{0}, \lambda\right)-\psi_{+}\left(z_{0}, \lambda\right) .
\end{gathered}
$$

These are the well-known conditions of continuity of the asymptotic solutions $\psi_{\mp}(z, \lambda)$ and of their first derivatives $\psi_{\mp}^{(1)}(z, \lambda)$ at the chosen boundary $z=z_{0}$. They correspond to the approximation of the genuine potential $u(z)$ by the abrupt transition of $u_{-}(z)$ into the potential $u_{+}(z)$ at the reference point $z=z_{0}$ (see Figure 1 and Figure 2).

The Sturm-Liouville equation provides for arbitrary fixed parameter $\lambda$ and asymptotic potentials $u_{-}(z)$ and $u_{+}(z)$ solutions $\psi_{-}(z, \lambda)$ and $\psi_{+}(z, \lambda)$, respectively, with the superposition of two possible basic solutions with 2 free amplitudes in each case and this total number of 4 amplitudes is related by the 2 boundary conditions to 2 free amplitudes (e.g., incident waves from each side of the boundary) and to 2 determinable amplitudes from them (e.g., for reflected or refracted waves in dependence from which side one sees this).

We now consider finite approximations to the boundary conditions (4.8) taking into account a transition region between the two asymptotic potentials with given properties. They have to be non-contradictory and each higher approximation should contain the preceding approximation by neglect of terms. In this way we can assume that in an infinite process which, clearly, is impossible to make every term in the boundary conditions will be taken into account.

\subsection{First-Order Approximation of the Boundary Conditions}

In the first-order approximation of the boundary conditions we consider the solution in the approximate form

$$
\psi(z, \lambda)=\psi_{-}(z, \lambda) \theta\left(z_{0}-z\right)+\psi_{+}(z, \lambda) \theta\left(z-z_{0}\right)+\psi_{0}^{\prime}\left(z_{0}, \lambda\right) \delta\left(z-z_{0}\right)
$$


and in addition we take into account the moments $u_{\mp, 0}\left(z_{0}\right)$ and neglect all higher moments, i.e. $u_{\mp, n}\left(z_{0}\right)=0,(n \geq 1)$.

Then we find from (4.1), (4.2) and (4.3) for $n=2$ the following boundary conditions

$$
\begin{gathered}
0=\psi_{-}^{(1)}\left(z_{0}, \lambda\right)-\psi_{+}^{(1)}\left(z_{0}, \lambda\right)+\left(u_{-, 0}\left(z_{0}\right) \psi_{-}\left(z_{0}, \lambda\right)\right. \\
\left.+u_{+, 0}\left(z_{0}\right) \psi_{+}\left(z_{0}, \lambda\right)+\left(u\left(z_{0}\right)-\lambda\right) \psi_{0}^{\prime}\left(z_{0}, \lambda\right)\right) \\
0=\psi_{-}\left(z_{0}, \lambda\right)-\psi_{+}\left(z_{0}, \lambda\right) \\
0=-\psi_{0}^{\prime}\left(z_{0}, \lambda\right) .
\end{gathered}
$$

Due to $\psi_{0}\left(z_{0}, \lambda\right)=0$ one obtains by its elimination from the first equation and using the equality $\psi_{-}\left(z_{0}, \lambda\right)=\psi_{+}\left(z_{0}, \lambda\right)$ effectively the following two boundary conditions in first approximation

$$
\begin{gathered}
0=\psi_{-}^{(1)}\left(z_{0}, \lambda\right)-\psi_{+}^{(1)}\left(z_{0}, \lambda\right)+\frac{u_{0}\left(z_{0}\right)}{2}\left(\psi_{-}\left(z_{0}, \lambda\right)+\psi_{+}\left(z_{0}, \lambda\right)\right), \\
0=\psi_{-}\left(z_{0}, \lambda\right)-\psi_{+}\left(z_{0}, \lambda\right) .
\end{gathered}
$$

In considered approximation the moments $u_{-, 0}\left(z_{0}\right)$ and $u_{+, 0}\left(z_{0}\right)$ are involved only as their sum $u_{-, 0}\left(z_{0}\right)+u_{+, 0}\left(z_{0}\right) \quad$ (see also Figure 1 and Figure 2)

$$
\begin{aligned}
u_{0}\left(z_{0}\right) & =u_{-, 0}\left(z_{0}\right)+u_{+, 0}\left(z_{0}\right) \\
& =\int_{-\infty}^{+\infty} \mathrm{d} z\left(u(z)-u_{-}(z) \theta\left(z_{0}-z\right)-u_{+}(z) \theta\left(z-z_{0}\right)\right) \\
& \equiv \int_{-\infty}^{+\infty} \mathrm{d} z \Delta u\left(z_{0} ; z\right) .
\end{aligned}
$$

A quantity such as $u_{-, 0}\left(z_{0}\right)+u_{+, 0}\left(z_{0}\right)$ and its dependence on the choice of $z_{0}$ in optics and its connection to higher such moments was discussed by Lekner in his versatile monograph [13] in chap. 3 (in particular p. 81, Figure 3.1 and Eq. (3.27)) and they were called integral invariants but its origin from moment-series expansion of function (see Equations (A.17) and (A.23) in Appendix $A)$ is not mentioned there. Such quantities played already a great role in our paper [12]. It became clear there that the functions $\left(u_{-}(z)-u(z)\right) \theta\left(z_{0}-z\right)$ and $\left(u_{+}(z)-u(z)\right) \theta\left(z-z_{0}\right)$ (correspondingly $\varepsilon(z ; \omega)$ in optics) and their moments have to be considered separately and that they usually are involved in higher-order approximations separately (see next subsections). In the old papers of Drude [1] [2] (see also [3] [5] [6]) moments of the permeability play also a role but the asymptotic parts are not split there and the theory of Generalized functions they did not have to disposal.

If the asymptotic potentials $u_{-}(z)$ and $u_{+}(z)$ are smooth (existence of all its derivatives) then by Taylor-series expansions of the step functions $\theta\left(z_{0}-z\right)$ and $\theta\left(z-z_{0}\right)$ in powers of $z_{0}$ and using formulae (A.5) and (A.2) one obtains the following expansion of $u_{0}\left(z_{0}\right)$ in powers of $z_{0}$

$$
\begin{aligned}
u_{0}\left(z_{0}\right) & =u_{0}(0)-\sum_{k=0}^{\infty}\left(u_{-}^{(k)}(0)-u_{+}^{(k)}(0)\right) \frac{z_{0}^{k+1}}{(k+1) !} \\
& =u_{0}(0)-\left(u_{-}(0)-u_{+}(0)\right) z_{0}-\left(u_{-}^{(1)}(0)-u_{+}^{(1)}(0)\right) \frac{z_{0}^{2}}{2}-\cdots
\end{aligned}
$$


A favorable choice of the reference point $z_{0}$ is often the condition $u_{0}\left(z_{0}\right)=0$, approximately

$$
0 \approx u_{0}(0)-\left(u_{-}(0)-u_{+}(0)\right) z_{0}, \quad \Rightarrow \quad z_{0} \approx \frac{u_{0}(0)}{u_{-}(0)-u_{+}(0)}
$$

if $u_{-}(0)-u_{+}(0)$ is non-vanishing. Then $u_{0}\left(z_{0}\right) \equiv u_{-, 0}\left(z_{0}\right)+u_{+, 0}\left(z_{0}\right)$ is nearly vanishing and this choice is unique but, generally, it can be not unique due to nonlinearity of (4.13) in $z_{0}$.

The only effect in this approximation in comparison to zeroth approximation is then an influence on the phase relations between the asymptotic solutions on both sides. In case of not monotonically increasing or decreasing transitions the choice of vanishing moment $u_{-, 0}\left(z_{0}\right)+u_{+, 0}\left(z_{0}\right)$ is not in every case possible and unique, in particular, in case of a potential barrier or well between two equal asymptotic potentials we cannot suppose a monotonic increase or decrease of transition between the two potentials and this case has to be dealt with separately (see Section 5).

The unique choice of a boundary $z_{0}$ by the condition (4.14) can be also applied under the discussed suppositions in the higher-order approximations with favorable influence on the smallness of the involved higher moments and we do not discuss this again in the next considered approximations.

\subsection{Second-Order Approximation of the Boundary Conditions}

In the second-order approximation of the boundary conditions we use for the solution the following proposition

$$
\begin{aligned}
\psi(z, \lambda)= & \psi_{-}(z, \lambda) \theta\left(z_{0}-z\right)+\psi_{+}(z, \lambda) \theta\left(z-z_{0}\right) \\
& +\psi_{0}^{\prime}\left(z_{0}, \lambda\right) \delta\left(z-z_{0}\right)+\psi_{1}^{\prime}\left(z_{0}, \lambda\right) \delta^{(1)}\left(z-z_{0}\right)
\end{aligned}
$$

and take into account as non-vanishing parameters the moments $u_{\mp, 0}\left(z_{0}\right)$ and $u_{\mp, 1}\left(z_{0}\right)$ and neglect all others, i.e. $u_{\mp, n}\left(z_{0}\right)=0,(n \geq 2)$. From (4.1) and (4.2) and from (4.3) for $n=2,3$ we find then

$$
\begin{gathered}
0=\psi_{-}^{(1)}\left(z_{0}, \lambda\right)-\psi_{+}^{(1)}\left(z_{0}, \lambda\right) \\
+\left(u_{-, 0}\left(z_{0}\right) \psi_{-}\left(z_{0}, \lambda\right)+u_{+, 0}\left(z_{0}\right) \psi_{+}\left(z_{0}, \lambda\right)+\left(u\left(z_{0}\right)-\lambda\right) \psi_{0}^{\prime}\left(z_{0}, \lambda\right)\right) \\
-\left(u_{-, 1}\left(z_{0}\right) \psi_{-}^{(1)}\left(z_{0}, \lambda\right)+u_{+, 1}\left(z_{0}\right) \psi_{+}^{(1)}\left(z_{0}, \lambda\right)+u^{(1)}\left(z_{0}\right) \psi_{1}^{\prime}\left(z_{0}, \lambda\right)\right) \\
0=\psi_{-}\left(z_{0}, \lambda\right)-\psi_{+}\left(z_{0}, \lambda\right)+\left(u_{-, 1}\left(z_{0}\right) \psi_{-}\left(z_{0}, \lambda\right)\right. \\
\left.+u_{+, 1}\left(z_{0}\right) \psi_{+}\left(z_{0}, \lambda\right)+\left(u\left(z_{0}\right)-\lambda\right) \psi_{1}^{\prime}\left(z_{0}, \lambda\right)\right) \\
0=-\psi_{0}^{\prime}\left(z_{0}, \lambda\right) \\
0=-\psi_{1}^{\prime}\left(z_{0}, \lambda\right) .
\end{gathered}
$$

Using the last two conditions of vanishing of $\psi_{0}^{\prime}\left(z_{0}, \lambda\right)$ and $\psi_{1}^{\prime}\left(z_{0}, \lambda\right)$ the boundary conditions in the second-order approximation can be reduced to the two conditions 


$$
\begin{aligned}
0= & \left(1-u_{-, 1}\left(z_{0}\right)\right) \psi_{-}^{(1)}\left(z_{0}, \lambda\right)-\left(1+u_{+, 1}\left(z_{0}\right)\right) \psi_{+}^{(1)}\left(z_{0}, \lambda\right) \\
& +\left(u_{-, 0}\left(z_{0}\right) \psi_{-}\left(z_{0}, \lambda\right)+u_{+, 0}\left(z_{0}\right) \psi_{+}\left(z_{0}, \lambda\right)\right), \\
0= & \left(1+u_{-, 1}\left(z_{0}\right)\right) \psi_{-}\left(z_{0}, \lambda\right)-\left(1-u_{+, 1}\left(z_{0}\right)\right) \psi_{+}\left(z_{0}, \lambda\right),
\end{aligned}
$$

and a favorable choice of the boundary $z_{0}$ is mostly possible by the condition (4.14).

\subsection{Third-Order Approximation of the Boundary Conditions}

In the third-order approximation of the boundary conditions we use the following proposition for the solution

$$
\begin{aligned}
\psi(z, \lambda)= & \psi_{-}(z, \lambda) \theta\left(z_{0}-z\right)+\psi_{+}(z, \lambda) \theta\left(z-z_{0}\right)+\psi_{0}^{\prime}\left(z_{0}, \lambda\right) \delta\left(z-z_{0}\right) \\
& +\psi_{1}^{\prime}\left(z_{0}, \lambda\right) \delta^{(1)}\left(z-z_{0}\right)+\psi_{2}^{\prime}\left(z_{0}, \lambda\right) \delta^{(2)}\left(z-z_{0}\right)
\end{aligned}
$$

and take into account as non-vanishing parameters the moments

$u_{\mp, 0}\left(z_{0}\right), u_{\mp, 1}\left(z_{0}\right)$ and $u_{\mp, 2}\left(z_{0}\right)$. In this approximation follows from (4.1) and (4.2) and from (4.3) for $n=2,3,4$

$$
\begin{aligned}
& 0= \psi_{-}^{(1)}\left(z_{0}, \lambda\right)-\psi_{+}^{(1)}\left(z_{0}, \lambda\right) \\
&+\left(u_{-, 0}\left(z_{0}\right) \psi_{-}\left(z_{0}, \lambda\right)+u_{+, 0}\left(z_{0}\right) \psi_{+}\left(z_{0}, \lambda\right)+\left(u\left(z_{0}\right)-\lambda\right) \psi_{0}^{\prime}\left(z_{0}, \lambda\right)\right) \\
&-\left(u_{-, 1}\left(z_{0}\right) \psi_{-}^{(1)}\left(z_{0}, \lambda\right)+u_{+, 1}\left(z_{0}\right) \psi_{+}^{(1)}\left(z_{0}, \lambda\right)+u^{(1)}\left(z_{0}\right) \psi_{1}^{\prime}\left(z_{0}, \lambda\right)\right) \\
&+\left(u_{-, 2}\left(z_{0}\right) \psi_{-}^{(2)}\left(z_{0}, \lambda\right)+u_{+, 2}\left(z_{0}\right) \psi_{+}^{(2)}\left(z_{0}, \lambda\right)+u^{(2)}\left(z_{0}\right) \psi_{2}^{\prime}\left(z_{0}, \lambda\right)\right), \\
& 0= \psi_{-}\left(z_{0}, \lambda\right)-\psi_{+}\left(z_{0}, \lambda\right) \\
&+\left(u_{-, 1}\left(z_{0}\right) \psi_{-}\left(z_{0}, \lambda\right)+u_{+, 1}\left(z_{0}\right) \psi_{+}\left(z_{0}, \lambda\right)+\left(u\left(z_{0}\right)-\lambda\right) \psi_{1}^{\prime}\left(z_{0}, \lambda\right)\right) \\
&-2\left(u_{-, 2}\left(z_{0}\right) \psi_{-}^{(1)}\left(z_{0}, \lambda\right)+u_{+, 2}\left(z_{0}\right) \psi_{+}^{(1)}\left(z_{0}, \lambda\right)+u^{(1)}\left(z_{0}\right) \psi_{2}^{\prime}\left(z_{0}, \lambda\right)\right), \\
& 0=\left(u_{-, 2}\left(z_{0}\right) \psi_{-}\left(z_{0}, \lambda\right)+u_{+, 2}\left(z_{0}\right) \psi_{+}\left(z_{0}, \lambda\right)+\left(u\left(z_{0}\right)-\lambda\right) \psi_{2}^{\prime}\left(z_{0}, \lambda\right)\right)-\psi_{0}^{\prime}\left(z_{0}, \lambda\right), 0=-\psi_{1}^{\prime}\left(z_{0}, \lambda\right), \\
& 0=-\psi_{2}^{\prime}\left(z_{0}, \lambda\right) .
\end{aligned}
$$

In these boundary conditions we have for the first time two new peculiarities. The first is the occurrence of the second-order derivatives $\psi_{\mp}^{(2)}\left(z_{0}, \lambda\right)$ of the asymptotic solutions at the boundary $z_{0}$. Due to the asymptotic Equations (3.2) one may substitute them according to

$$
\psi_{\mp}^{(2)}\left(z_{0}, \lambda\right)=\left(u_{\mp}\left(z_{0}\right)-\lambda\right) \psi_{\mp}\left(z_{0}, \lambda\right) .
$$

The second is the non-vanishing of the coefficients $\psi_{0}^{\prime}\left(z_{0}, \lambda\right)$ for the first time. Taking into account the vanishing of $\psi_{2}\left(z_{0}, \lambda\right)$ we can express them by

$$
\psi_{0}^{\prime}\left(z_{0}, \lambda\right)=u_{-, 2}\left(z_{0}\right) \psi_{-}\left(z_{0}, \lambda\right)+u_{+, 2}\left(z_{0}\right) \psi_{+}\left(z_{0}, \lambda\right) .
$$

Inserting this into (4.19) we obtain the following two effective boundary conditions

$$
\begin{aligned}
0= & \left(1-u_{-, 1}\left(z_{0}\right)\right) \psi_{-}^{(1)}\left(z_{0}, \lambda\right)-\left(1+u_{+, 1}\left(z_{0}\right)\right) \psi_{+}^{(1)}\left(z_{0}, \lambda\right) \\
& +\left(u_{-, 0}\left(z_{0}\right)+\left(u\left(z_{0}\right)+u_{-}\left(z_{0}\right)-2 \lambda\right) u_{-, 2}\left(z_{0}\right)\right) \psi_{-}\left(z_{0}, \lambda\right) \\
& +\left(u_{+, 0}\left(z_{0}\right)+\left(u\left(z_{0}\right)+u_{+}\left(z_{0}\right)-2 \lambda\right) u_{+, 2}\left(z_{0}\right)\right) \psi_{+}\left(z_{0}, \lambda\right),
\end{aligned}
$$




$$
\begin{aligned}
0= & \left(1+u_{-, 1}\left(z_{0}\right)\right) \psi_{-}\left(z_{0}, \lambda\right)-\left(1-u_{+, 1}\left(z_{0}\right)\right) \psi_{+}\left(z_{0}, \lambda\right) \\
& -2\left(u_{-, 2}\left(z_{0}\right) \psi_{-}^{(1)}\left(z_{0}, \lambda\right)+u_{+, 2}\left(z_{0}\right) \psi_{+}^{(1)}\left(z_{0}, \lambda\right)\right) .
\end{aligned}
$$

These are two boundary conditions for the asymptotic solutions $\psi_{\mp}(z, \lambda)$ taken for $z=z_{0}$ at the boundary, i.e., for $\psi_{\mp}\left(z_{0}, \lambda\right)$ and their first derivatives $\psi_{\mp}^{(1)}\left(z_{0}, \lambda\right)$ at the boundary with the moments $u_{\mp, 0}\left(z_{0}\right), u_{\mp, 1}\left(z_{0}\right), u_{\mp, 2}\left(z_{0}\right)$. The quantities $u\left(z_{0}\right), u_{\mp}\left(z_{0}\right)$ involved have to be considered as known or as calculable parameters from the potential $u(z)$. The mentioned peculiarities with the additional Equation (4.21) for $\psi_{0}^{\prime}\left(z_{0}, \lambda\right)$ are the proper reason that we explicitly wrote down the already complicated boundary conditions (4.22).

Higher-order approximations of the boundary condition than the considered third-order approximation become much more difficult since in these cases one has to insert also higher- than second-order derivatives of the asymptotic potentials $\psi_{\mp}(z, \lambda)$ at the boundary $z=z_{0}$. Using (3.2) they can be expressed by zeroth- and first-order derivatives of $\psi_{\mp}(z, \lambda)$ plus derivatives of $u_{\mp}(z)$ that, in principle, is makable but becomes very complicated.

In many cases it is possible to make further simplifications of the boundary conditions if we make suppositions about the symmetry properties of the deviations $\Delta u\left(z_{0} ; z\right)$ of the potentials in the transition regions defined in (3.10) in connection with assumptions of monotonic increasing or decreasing.

\section{Reflection and Refraction at Transition Layers between Two Asymptotically Constant Potentials in Second-Order Approximation}

For the illustration of the derived boundary conditions and as important application we consider now problems of reflection and refraction for incident waves at a transition layer. Our notations for the amplitudes of the corresponding waves are schematically shown in Figure 5.

One of the simplest asymptotic potentials are potentials which go rapidly to constant values $u_{-}(z) \rightarrow u_{-}$as $z \rightarrow-\infty$ and to $u_{+}(z) \rightarrow u_{+}$as $z \rightarrow+\infty$ and, therefore, where the deviations $\Delta u\left(z_{0} ; z\right)$ from these potentials are rapidly vanishing for $z \rightarrow \pm \infty$ in the inner region. The lengths in the inner region where the potential changes essentially must be small in comparison to characteristic lengths of the problem (e.g., wavelengths). Similar problems are sometimes calculated for concrete transition potentials, in case of potential barriers or potential wells for rectangular potentials. The WKB-approximation where the lengths of changes of the potentials are large in comparison to the characteristic lengths of the problem is the opposite case to our calculations (e.g., [20]).

Thus we consider now the following asymptotic potentials $u_{-}(z)$ and $u_{+}(z)$

$$
u_{-}(z \rightarrow-\infty) \equiv u_{-}, \quad u_{+}(z \rightarrow-\infty) \equiv u_{+} .
$$

Only solutions with the same value of the parameter $\lambda$ are coupled at the boundary. The general asymptotic solutions of Equation (3.2) for the constant 
Amplitudes of incident (i) and reflected or refracted ( $r$ ) waves

$\mathrm{u}(\mathrm{z})$

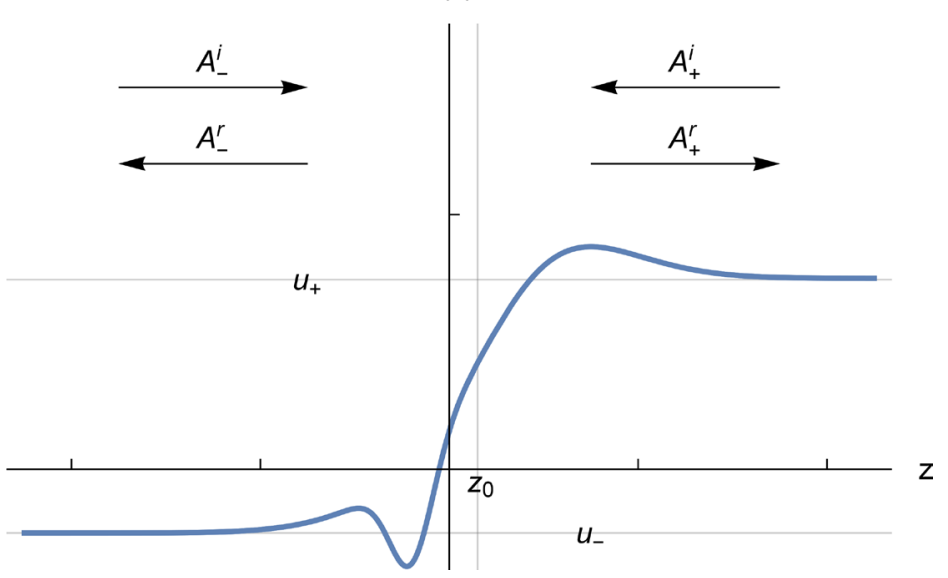

The upper indices " $i$ " and " $r$ " mean "incident" and "reflected or refracted", correspondingly. The lower indices "-" and "+" are chosen to see in equations which part on the coordinate axis is meant.

Figure 5. Notations of amplitudes of incident and reflected or refracted waves.

asymptotic potentials $u_{\mp}$ for each value of the parameter $\lambda$ are superpositions of two independent basic solutions and we set

$$
\begin{aligned}
& \psi_{-}(z, \lambda)=A_{-}^{\mathrm{i}} \mathrm{e}^{\mathrm{i} k_{-} z}+A_{-}^{\mathrm{r}} \mathrm{e}^{-\mathrm{i} k_{-} z}, \\
& \psi_{+}(z, \lambda)=A_{+}^{\mathrm{i}} \mathrm{e}^{-\mathrm{i} k_{+} z}+A_{+}^{\mathrm{r}} \mathrm{e}^{\mathrm{i} k_{+} z},
\end{aligned}
$$

with the dispersion relations (in principle, also true for complex values $\lambda$ )

$$
\begin{aligned}
& k_{-}=\sqrt{\lambda-u_{-}}, \quad k_{+}=\sqrt{\lambda-u_{+}}, \\
& \Leftrightarrow \lambda=k_{-}^{2}+u_{-}=k_{+}^{2}+u_{+}, \quad u_{-}-u_{+}=k_{+}^{2}-k_{-}^{2} .
\end{aligned}
$$

The complex amplitudes of the two possible waves on each side of the boundary $z=z_{0}$ are denoted by $A_{-}^{\mathrm{i}, \mathrm{r}}$ and $A_{+}^{\mathrm{i}, \mathrm{r}}$ (upper index " $\mathrm{i}$ " stands for "incident" and upper index " $r$ " for "reflected" or "refracted" in dependence on the side from which the incident and reflected or refracted waves are considered but only for real $\lambda$ and then chosen $k_{-}>0$ and $k_{+}>0$ these notations are uniquely justified). From (5.2) follows for the derivatives of the solutions

$$
\begin{aligned}
& \psi_{-}^{(1)}(z, \lambda)=+\mathrm{i} k_{-}\left(A_{-}^{\mathrm{i}} \mathrm{e}^{\mathrm{i} k_{-} z}-A_{-}^{\mathrm{r}} \mathrm{e}^{-\mathrm{i} k_{-} z}\right), \\
& \psi_{+}^{(1)}(z, \lambda)=-\mathrm{i} k_{+}\left(A_{+}^{\mathrm{i}} \mathrm{e}^{-\mathrm{i} k_{+} z}-A_{+}^{\mathrm{r}} \mathrm{e}^{\mathrm{i} k_{+} z}\right) .
\end{aligned}
$$

For $\lambda \geq u_{\mp}$ both solutions $k_{\mp}$ possess real values and denote then wavelengths and for $\lambda \leq u_{\mp}$ both solutions $k_{\mp}$ possess imaginary values and denote decay constants. For $u_{-}<\lambda<u_{+}$or $u_{-}>\lambda>u_{+}$only one of the parameters $k_{\mp}$ is real and the other $k_{ \pm}$is imaginary, respectively.

The derived boundary conditions at $z=z_{0}$ in arbitrary approximation can be written in form of a matrix equation, for example, in the following way

$$
\mathrm{S}^{\mathrm{i}}\left(\begin{array}{l}
A_{-}^{\mathrm{i}} \mathrm{e}^{+\mathrm{i} k_{-} z_{0}} \\
A_{+}^{\mathrm{i}} \mathrm{e}^{-\mathrm{i} k_{+} z_{0}}
\end{array}\right)=\mathrm{S}^{\mathrm{r}}\left(\begin{array}{c}
A_{-}^{\mathrm{r}} \mathrm{e}^{-\mathrm{i} k_{-} z_{0}} \\
A_{+}^{\mathrm{r}} \mathrm{e}^{+\mathrm{i} k_{+} z_{0}}
\end{array}\right),
$$

where $S^{i, r}$ are matrices depending on the considered approximations. These 
matrices $\mathrm{S}^{\mathrm{i}, \mathrm{r}}$ (or $\mathrm{S}_{\mp}$ ) become more complicated with each higher approximation of the boundary conditions and the jump from the second approximation (4.17) to the third approximation (4.22) is already very large and leads to complicated expression.

In the second-order approximation (4.17) we find for the considered asymptotically constant potentials the following matrices in case of (5.9)

$$
\begin{aligned}
& S^{\mathrm{i}}=\left(\begin{array}{cc}
\left(1-u_{-, 1}\left(z_{0}\right)\right) k_{-}-\mathrm{i} u_{-, 0}\left(z_{0}\right) & \left(1+u_{+, 1}\left(z_{0}\right)\right) k_{+}-\mathrm{i} u_{+, 0}\left(z_{0}\right) \\
1+u_{-, 1}\left(z_{0}\right) & -1+u_{+, 1}\left(z_{0}\right)
\end{array}\right), \\
& S^{\mathrm{r}}=\left(\begin{array}{cc}
\left(1-u_{-, 1}\left(z_{0}\right)\right) k_{-}+\mathrm{i} u_{-, 0}\left(z_{0}\right) & \left(1+u_{+, 1}\left(z_{0}\right)\right) k_{+}+\mathrm{i} u_{+, 0}\left(z_{0}\right) \\
-1-u_{-, 1}\left(z_{0}\right) & 1-u_{+, 1}\left(z_{0}\right)
\end{array}\right) .
\end{aligned}
$$

Due to two-dimensional Hamilton-Cayley identity of an arbitrary two-dimensional matrix $\mathrm{S}$

$$
\mathrm{S}^{2}-\langle\mathrm{S}\rangle \mathrm{S}+[\mathrm{S}] \mathrm{I}=0, \quad[\mathrm{~S}] \equiv \frac{1}{2}\left(\langle\mathrm{~S}\rangle^{2}-\left\langle\mathrm{S}^{2}\right\rangle\right),
$$

where $\langle S\rangle$ is the trace and [S] the determinant of $S$ and I denotes the identity matrix one finds the inverse matrix $\mathrm{S}^{-1}$ in coordinate-invariant way by ${ }^{4}$

$$
\overline{\mathrm{S}} \equiv\langle\mathrm{S}\rangle \mathrm{I}-\mathrm{S}, \quad \Rightarrow\langle\overline{\mathrm{S}}\rangle=\langle\mathrm{S}\rangle, \quad[\overline{\mathrm{S}}]=[\mathrm{S}], \quad \overline{\mathrm{S}} \mathrm{S}=[\mathrm{S}] \mathrm{I}, \quad \mathrm{S}^{-1}=\frac{\overline{\mathrm{S}}}{[\mathrm{S}]},
$$

where $\bar{S}$ by definition denotes the associated matrix to the matrix $\mathrm{S}$.

By multiplication of (5.6) with the inverse matrix $\left(S^{r}\right)^{-1}$ to $S^{r}$ one may resolve the equations to the reflected or refracted wave amplitudes in dependence on the amplitudes of the incident waves.

$$
\left(\begin{array}{l}
A_{-}^{\mathrm{r}} \mathrm{e}^{-\mathrm{i} k_{-} z_{0}} \\
A_{+}^{\mathrm{r}} \mathrm{e}^{\mathrm{i} k_{+} z_{0}}
\end{array}\right)=\frac{\overline{\mathrm{S}^{\mathrm{r}}} \mathrm{S}^{\mathrm{i}}}{\left[\mathrm{S}^{\mathrm{r}}\right]}\left(\begin{array}{l}
A_{-}^{\mathrm{i}} \mathrm{e}^{+\mathrm{i} k_{-} z_{0}} \\
A_{+}^{\mathrm{i}} \mathrm{e}^{-\mathrm{i} k_{+} z_{0}}
\end{array}\right) \equiv \mathrm{R}\left(\begin{array}{l}
A_{-}^{\mathrm{i}} \mathrm{e}^{+\mathrm{i} k_{-} z_{0}} \\
A_{+}^{\mathrm{i}} \mathrm{e}^{-\mathrm{i} k_{+} z_{0}}
\end{array}\right)=\left(\begin{array}{cc}
R_{--} & R_{-+} \\
R_{+-} & R_{++}
\end{array}\right)\left(\begin{array}{l}
A_{-}^{\mathrm{i}} \mathrm{e}^{+\mathrm{i} k_{-} z_{0}} \\
A_{+}^{\mathrm{i}} \mathrm{e}^{-\mathrm{i} k_{+} z_{0}}
\end{array}\right) .
$$

We find for the numerators of the matrix elements of the reflection-refraction matrix $\mathrm{R}$

$$
\begin{gathered}
{\left[\mathrm{S}^{\mathrm{r}}\right] R_{--}=\left(1+u_{-, 1}\left(z_{0}\right) u_{+, 1}\left(z_{0}\right)\right)\left(k_{-}-k_{+}\right)-\left(u_{-, 1}\left(z_{0}\right)+u_{+, 1}\left(z_{0}\right)\right)\left(k_{-}+k_{+}\right)} \\
-\mathrm{i}\left(u_{-, 0}\left(z_{0}\right)+u_{+, 0}\left(z_{0}\right)-u_{-, 0}\left(z_{0}\right) u_{+, 1}\left(z_{0}\right)+u_{+, 0}\left(z_{0}\right) u_{-, 1}\left(z_{0}\right)\right), \\
{\left[\mathrm{S}^{\mathrm{r}}\right] R_{-+}=2\left(1-u_{+, 1}^{2}\left(z_{0}\right)\right) k_{+},} \\
{\left[\mathrm{S}^{\mathrm{r}}\right] R_{+-}=2\left(1-u_{-, 1}^{2}\left(z_{0}\right)\right) k_{-},} \\
{\left[\mathrm{S}^{\mathrm{r}}\right] R_{++}=-\left(1+u_{-, 1}\left(z_{0}\right) u_{+, 1}\left(z_{0}\right)\right)\left(k_{-}-k_{+}\right)+\left(u_{-, 1}\left(z_{0}\right)+u_{+, 1}\left(z_{0}\right)\right)\left(k_{-}+k_{+}\right)} \\
-\mathrm{i}\left(u_{-, 0}\left(z_{0}\right)+u_{+, 0}\left(z_{0}\right)-u_{-, 0}\left(z_{0}\right) u_{+, 1}\left(z_{0}\right)+u_{+, 0}\left(z_{0}\right) u_{-, 1}\left(z_{0}\right)\right),
\end{gathered}
$$

and for the determinant of the matrix $S^{r}$

${ }^{4}$ Coordinate-invariant calculations in 2D-case usually do not bring a great advantage in comparison to coordinate calculations due to the relatively simple formulae

$$
\mathrm{S} \equiv\left(\begin{array}{ll}
\mathrm{S}_{11}, & \mathrm{~S}_{12} \\
\mathrm{~S}_{21}, & \mathrm{~S}_{22}
\end{array}\right), \quad \overline{\mathrm{S}} \equiv\left(\begin{array}{cc}
\mathrm{S}_{22}, & -\mathrm{S}_{12} \\
-\mathrm{S}_{21}, & \mathrm{~S}_{11}
\end{array}\right), \quad\langle\mathrm{S}\rangle \equiv \mathrm{S}_{11}+\mathrm{S}_{22}, \quad[\mathrm{~S}] \equiv \mathrm{S}_{11} \mathrm{~S}_{22}-\mathrm{S}_{12} \mathrm{~S}_{21} .
$$

However, already in 3D-case the advantage is mostly important and we prefer them everywhere. 


$$
\begin{aligned}
{\left[\mathrm{S}^{\mathrm{r}}\right]=} & \left(1+u_{-, 1}\left(z_{0}\right) u_{+, 1}\left(z_{0}\right)\right)\left(k_{-}+k_{+}\right)-\left(u_{-, 1}\left(z_{0}\right)+u_{+, 1}\left(z_{0}\right)\right)\left(k_{-}-k_{+}\right) \\
& +\mathrm{i}\left(u_{-, 0}\left(z_{0}\right)+u_{+, 0}\left(z_{0}\right)-u_{-, 0}\left(z_{0}\right) u_{+, 1}\left(z_{0}\right)+u_{+, 0}\left(z_{0}\right) u_{-, 1}\left(z_{0}\right)\right) .
\end{aligned}
$$

Already into this second-order approximation the moments $u_{-, v}\left(z_{0}\right)$ and $u_{+, v}\left(z_{0}\right)$ are separately involved and not only as their sums

$u_{-, v}\left(z_{0}\right)+u_{+, v}\left(z_{0}\right),(v=0,1)$. The formulae (5.9) together with (5.10) and (5.11) describe the already complicated second-order approximation of reflection and refraction at a transition layer between two asymptotically constant potentials $u_{-}$and $u_{+}$which are contained in the expressions (5.3) for $k_{-}$and $k_{+}$.

\section{First-Order Approximation of Reflection and Refraction Formulae and Their Invariance with Small Variation of Boundary Choice}

In first-order approximation we neglect the moments $u_{\mp, 1}\left(z_{0}\right)$ and obtain from (5.10) and (5.11) the following formulae for the amplitudes of the reflected and refracted waves in dependence on the amplitudes of the incident waves

$$
\left(\begin{array}{l}
A_{-}^{\mathrm{r}} \mathrm{e}^{-\mathrm{i} k_{-} z_{0}} \\
A_{+}^{\mathrm{r}} \mathrm{e}^{+\mathrm{i} k_{+} z_{0}}
\end{array}\right)=\left(\begin{array}{cc}
\frac{k_{-}-k_{+}-\mathrm{i} u_{0}\left(z_{0}\right)}{k_{-}+k_{+}+\mathrm{i} u_{0}\left(z_{0}\right)} & \frac{2 k_{+}}{k_{-}+k_{+}+\mathrm{i} u_{0}\left(z_{0}\right)} \\
\frac{2 k_{-}}{k_{-}+k_{+}+\mathrm{i} u_{0}\left(z_{0}\right)} & \frac{k_{+}-k_{-}-\mathrm{i} u_{0}\left(z_{0}\right)}{k_{-}+k_{+}+\mathrm{i} u_{0}\left(z_{0}\right)}
\end{array}\right)\left(\begin{array}{l}
A_{-}^{\mathrm{i}} \mathrm{e}^{+\mathrm{i} k_{-} z_{0}} \\
A_{+}^{\mathrm{i}} \mathrm{e}^{-\mathrm{i} k_{+} z_{0}}
\end{array}\right),
$$

with the inversion

$$
\left(\begin{array}{l}
A_{-}^{\mathrm{i}} \mathrm{e}^{+\mathrm{i} k_{-} z_{0}} \\
A_{+}^{\mathrm{i}} \mathrm{e}^{-\mathrm{i} k_{+} z_{0}}
\end{array}\right)=\left(\begin{array}{cc}
\frac{k_{-}-k_{+}+\mathrm{i} u_{0}\left(z_{0}\right)}{k_{-}+k_{+}-\mathrm{i} u_{0}\left(z_{0}\right)} & \frac{2 k_{+}}{k_{-}+k_{+}-\mathrm{i} u_{0}\left(z_{0}\right)} \\
\frac{2 k_{-}}{k_{-}+k_{+}-\mathrm{i} u_{0}\left(z_{0}\right)} & \frac{k_{+}-k_{-}+\mathrm{i} u_{0}\left(z_{0}\right)}{k_{-}+k_{+}-\mathrm{i} u_{0}\left(z_{0}\right)}
\end{array}\right)\left(\begin{array}{l}
A_{-}^{\mathrm{r}} \mathrm{e}^{-\mathrm{i} k_{-} z_{0}} \\
A_{+}^{\mathrm{r}} \mathrm{e}^{+\mathrm{i} k_{+} z_{0}}
\end{array}\right) .
$$

The moment $u_{0}\left(z_{0}\right)$ is the sum of the moments $u_{-, 0}\left(z_{0}\right)$ and $u_{+, 0}\left(z_{0}\right)$ and corresponds to the integral over the deviation of the genuine potential from the asymptotic potentials (see Figure 1 and Figure 2)

$$
\begin{aligned}
u_{0}\left(z_{0}\right) & \equiv u_{-, 0}\left(z_{0}\right)+u_{+, 0}\left(z_{0}\right) \\
& =\int_{-\infty}^{+\infty} \mathrm{d} z\left(u(z)-u_{-} \theta\left(z_{0}-z\right)-u_{+} \theta\left(z-z_{0}\right)\right) .
\end{aligned}
$$

Due to constant asymptotic potentials its Taylor series in powers of $z_{0}$ possesses only two terms (see also (4.13))

$$
u_{0}\left(z_{0}\right)=u_{0}(0)-\left(u_{-}-u_{+}\right) z_{0}=u_{0}(0)-\left(k_{+}^{2}-k_{-}^{2}\right) z_{0},
$$

where (5.3) is taken into account. For $u_{-}-u_{+} \neq 0$ (genuine transition between asymptotically different potentials) the reference point $z_{0}$ of the expansions (the "boundary") can be uniquely chosen in such way that the moment $u_{0}\left(z_{0}\right)$ vanishes leading to

$$
u_{0}\left(z_{0}\right)=0, \Rightarrow z_{0}=\frac{u_{0}(0)}{u_{-}-u_{+}}=\frac{u_{0}(0)}{k_{+}^{2}-k_{-}^{2}} .
$$

This is likely the "best" choice of the point $z_{0}$ since in this case the matrix of ref- 
lection and refraction takes on the usual form without a transition layer and the presence of the last is then involved in the additional phase factors at the amplitudes in (6.1) in comparison to the usual formulae in case of step-wise (abrupt) transition at $z_{0}=0$. Formally, with substitutions they are even identical with the relations between the amplitudes of the electric field of incident, reflected and refracted waves for oblique incidence when the electric field is polarized perpendicular to the incidence plane (s-waves; see [14], chap. X, section 86, Eqs. (86.4)).

The matrix Equation (6.1) can be also represented in the form

$$
\left(\begin{array}{l}
A_{-}^{\mathrm{r}} \\
A_{+}^{\mathrm{r}}
\end{array}\right)=\left(\begin{array}{ll}
\frac{k_{-}-k_{+}-\mathrm{i} u_{0}\left(z_{0}\right)}{k_{-}+k_{+}+\mathrm{i} u_{0}\left(z_{0}\right)} \mathrm{e}^{+\mathrm{i} 2 k_{-} z_{0}} & \frac{2 k_{+}}{k_{-}+k_{+}+\mathrm{i} u_{0}\left(z_{0}\right)} \mathrm{e}^{+\mathrm{i}\left(k_{-}-k_{+}\right) z_{0}} \\
\frac{2 k_{-}}{k_{-}+k_{+}+\mathrm{i} u_{0}\left(z_{0}\right)} \mathrm{e}^{+\mathrm{i}\left(k_{-}-k_{+}\right) z_{0}} & \frac{k_{+}-k_{-}-\mathrm{i} u_{0}\left(z_{0}\right)}{k_{-}+k_{+}+\mathrm{i} u_{0}\left(z_{0}\right)} \mathrm{e}^{-\mathrm{i} 2 k_{+} z_{0}}
\end{array}\right)\left(\begin{array}{c}
A_{-}^{\mathrm{i}} \\
A_{+}^{\mathrm{i}}
\end{array}\right) .
$$

We show now that the matrix in (6.6) which determines the relation between the amplitudes of the incident and reflected waves is nearly unsensible against small variations of the boundary $z_{0}$. For example, for the first matrix element in (6.6) we have

$$
\begin{aligned}
& \frac{k_{-}-k_{+}-\mathrm{i} u_{0}\left(z_{0}\right)}{k_{-}+k_{+}+\mathrm{i} u_{0}\left(z_{0}\right)} \mathrm{e}^{+\mathrm{i} 2 k_{-} z_{0}}=\frac{\left\{k_{-}-k_{+}-\mathrm{i} u_{0}\left(z_{0}\right)\right\} \mathrm{e}^{\mathrm{i}\left(k_{-}+k_{+}\right) z_{0}}}{\left\{k_{-}+k_{+}+\mathrm{i} u_{0}\left(z_{0}\right)\right\} \mathrm{e}^{-\mathrm{i}\left(k_{-}-k_{+} z_{0}\right)}} \\
& \approx \frac{\left\{k_{-}-k_{+}-\mathrm{i}\left(u_{0}(0)-\left(u_{-}-u_{+}\right) z_{0}\right)\right\}\left(1+\mathrm{i}\left(k_{-}+k_{+}\right) z_{0}\right)}{\left\{k_{-}+k_{+}+\mathrm{i}\left(u_{0}(0)-\left(u_{-}-u_{+}\right) z_{0}\right)\right\}\left(1-\mathrm{i}\left(k_{-}-k_{+}\right) z_{0}\right)} \\
& \approx \frac{k_{-}-k_{+}-\mathrm{i} u_{0}(0)+\mathrm{i}\left(u_{-}-u_{+}+\left(k_{-}^{2}-k_{+}^{2}\right)\right) z_{0}}{k_{-}+k_{+}+\mathrm{i} u_{0}(0)-\mathrm{i}\left(u_{-}-u_{+}+\left(k_{-}^{2}-k_{+}^{2}\right)\right) z_{0}}=\frac{k_{-}-k_{+}-\mathrm{i} u_{0}(0)}{k_{-}+k_{+}+\mathrm{i} u_{0}(0)},
\end{aligned}
$$

due to (5.3) and where we neglected sum terms proportional to $z_{0}^{2}$ in numerator and denominator as "small" quantities. In full analogy we may treat the other matrix elements where in the non-diagonal elements we have only to transform the denominators. Thus if $z=z_{0}$ is already a "good" approximation for the boundary we may simplify (6.6) to

$$
\left(\begin{array}{c}
A_{-}^{\mathrm{r}} \\
A_{+}^{\mathrm{r}}
\end{array}\right)=\left(\begin{array}{cc}
\frac{k_{-}-k_{+}-\mathrm{i} u_{0}(0)}{k_{-}+k_{+}+\mathrm{i} u_{0}(0)} & \frac{2 k_{+}}{k_{-}+k_{+}+\mathrm{i} u_{0}(0)} \\
\frac{2 k_{-}}{k_{-}+k_{+}+\mathrm{i} u_{0}(0)} & \frac{k_{+}-k_{-}-\mathrm{i} u_{0}(0)}{k_{-}+k_{+}+\mathrm{i} u_{0}(0)}
\end{array}\right)\left(\begin{array}{c}
A_{-}^{\mathrm{i}} \\
A_{+}^{\mathrm{i}}
\end{array}\right) .
$$

In view of the complexity of the second-order approximation of the reflection and refraction formulae (5.9), (5.10) and (5.11) we did not check them in analogous way with respect to their approximate invariance under small variations of the reference point $z_{0}$.

\section{Semi-Bound and Bound States for Asymptotically of Constant Potentials}

The formulae of the preceding Sections 5 and 6 are true, in principle, for complex values $\lambda$ in connection with (5.2) and (5.3). 
For real values $\lambda$ in cases of $\lambda<u_{-}$or $\lambda<u_{+}$we find according to (5.3) imaginary values of $k_{-}$or $k_{+}$and we set in these special cases, respectively

$$
k_{-} \rightarrow \mathrm{i} \kappa_{-}, \quad k_{+} \rightarrow \mathrm{i} \kappa_{+},
$$

corresponding to exponentially decreasing or increasing factors for $z \rightarrow \mp \infty$. From these factors only $\mathrm{e}^{+\kappa_{-} z}$ for $z \rightarrow-\infty$ and $\mathrm{e}^{-\kappa_{+} z}$ for $z \rightarrow+\infty$ may correspond to finite sources in "infinity". This implies that in addition to the considered general case we have to distinguish interesting special cases with exponential vanishing of the solutions in infinity. If we concretely assume now $u_{-} \leq u_{+}$, we have first the case of semi-bound states

$$
\begin{gathered}
u_{-} \leq \lambda \leq u_{+}, \\
k_{-} \equiv \sqrt{\lambda-u_{-}}, \quad \kappa_{+} \equiv-\mathrm{i} k_{+}=\sqrt{u_{+}-\lambda} \geq 0,
\end{gathered}
$$

with the form of the solutions (we set $A_{+}^{\mathrm{i}}=0, A_{+}^{\mathrm{r}} \equiv A_{+}$)

$$
\psi_{-}(\lambda, z)=A_{-}^{\mathrm{i}} \mathrm{e}^{+\mathrm{i} k_{-} z}+A_{-}^{\mathrm{r}} \mathrm{e}^{-\mathrm{i} k_{-} z}, \quad \psi_{+}(z, \lambda)=A_{+} \mathrm{e}^{-\kappa_{+} z} .
$$

The second interesting case concerns bound states

$$
\begin{gathered}
\lambda \leq u_{-} \leq u_{+}, \\
\kappa_{-} \equiv-\mathrm{i} k_{-}=\sqrt{u_{-}-\lambda} \geq 0, \quad \kappa_{+} \equiv-\mathrm{i} k_{+}=\sqrt{u_{+}-\lambda} \geq 0,
\end{gathered}
$$

with the form of the solutions (we set $A_{-}^{\mathrm{i}}=A_{+}^{\mathrm{i}}=0, A_{-}^{\mathrm{r}} \equiv A_{-}, A_{+}^{\mathrm{r}} \equiv A_{+}$)

$$
\psi_{-}(\lambda, z)=A_{-} \mathrm{e}^{+\kappa_{-} z}, \quad \psi_{+}(\lambda, z)=A_{+} \mathrm{e}^{-\kappa_{+} z} .
$$

In the semi-bound case in the first-order approximation of the boundary conditions we find from (6.1) for the amplitudes of the reflected wave $A_{-}^{\mathrm{r}}$ on the negative side and the exponentially decreasing wave $A_{+}$on the positive side in dependence on the amplitude of the incident wave $A_{-}^{\mathrm{i}}$ on the negative side and in dependence on the reference point $z_{0}$

$$
\begin{aligned}
& A_{-}^{\mathrm{r}} \mathrm{e}^{-\mathrm{i} k_{-} z_{0}}=\frac{k_{-}-\mathrm{i} \kappa_{+}-\mathrm{i} u_{0}\left(z_{0}\right)}{k_{-}+\mathrm{i} \kappa_{+}+\mathrm{i} u_{0}\left(z_{0}\right)} A_{-}^{\mathrm{i}} \mathrm{e}^{\mathrm{i} k_{-} z_{0}}, \\
& A_{+} \mathrm{e}^{-\kappa_{+} z_{0}}=\frac{2 k_{-}}{k_{-}+\mathrm{i} \kappa_{+}+\mathrm{i} u_{0}\left(z_{0}\right)} A_{-}^{\mathrm{i}} \mathrm{e}^{\mathrm{i} k_{-} z_{0}},
\end{aligned}
$$

with

$$
k_{-} \equiv \sqrt{\lambda-u_{-}} \geq 0, \quad \kappa_{+} \equiv \sqrt{u_{+}-\lambda} \geq 0 .
$$

Semi-bound states as seen from (7.2) exist as solutions of the Sturm-Liouville equation for asymptotically constant potentials for a whole band of values $\lambda$ in the range $u_{-} \leq \lambda \leq u_{+}$and therefore are not discrete states. They are non-degenerate that means for each possible value $\lambda$ in the given range exists one state. In some aspects they are analogous to total reflection in optics.

We come now to bound states which are characterized by the condition (7.4). The dispersion equation can be obtained from vanishing of the denominators in the matrix elements of in (6.1) but the amplitude relation are difficult to find directly from them. Therefore we go back for the case $\lambda \leq u_{-} \leq u_{+}$directly to the 
boundary relations in first-order approximation with the proposition for the solution

$$
\begin{array}{ll}
\psi_{-}(z, \lambda)=A_{-} \mathrm{e}^{+\kappa_{-} z}, & \psi_{-}^{(1)}(z, \lambda)=+\kappa_{-} A_{-} \mathrm{e}^{+\kappa_{-} z}, \\
\psi_{+}(z, \lambda)=A_{+} \mathrm{e}^{-\kappa_{+} z}, \quad \psi_{+}^{(1)}(z, \lambda)=-\kappa_{-} A_{+} \mathrm{e}^{-\kappa_{+} z},
\end{array}
$$

with the relations (7.4)

$$
\kappa_{+}=\sqrt{u_{+}-\lambda} \geq \kappa_{-}=\sqrt{u_{-}-\lambda} \geq 0 .
$$

Inserting (7.8) into the boundary conditions (4.11) we find written in matrix form

$$
0=\left(\begin{array}{cc}
\kappa_{-}+\frac{u_{0}\left(z_{0}\right)}{2} & \kappa_{+}+\frac{u_{0}\left(z_{0}\right)}{2} \\
-1 & 1
\end{array}\right)\left(\begin{array}{l}
A_{-} \mathrm{e}^{+\kappa_{-} z_{0}} \\
A_{+} \mathrm{e}^{-\kappa_{+} z_{0}}
\end{array}\right) .
$$

Condition for the existence of non-trivial solutions is the vanishing of the determinant of the matrix in (7.10), i.e.

$$
0=\kappa_{-}+\kappa_{+}+u_{0}\left(z_{0}\right), \quad \Rightarrow \quad u_{0}\left(z_{0}\right)=-\kappa_{-}-\kappa_{+} \leq 0,
$$

and for the amplitude relations using (7.11) we obtain

$$
A_{-} \mathrm{e}^{+\kappa_{-} z_{0}}=A_{+} \mathrm{e}^{-\kappa_{+} z_{0}} \text {. }
$$

We now resolve the dispersion Equation (7.11) with respect to $\lambda$ using (7.9). Squaring $\kappa_{+}+\kappa_{-}$from (7.11) follows

$$
u_{0}^{2}\left(z_{0}\right)-\left(\kappa_{+}^{2}+\kappa_{-}^{2}\right)=2 \kappa_{+} \kappa_{-} .
$$

Squaring both sides of this equation again we arrive at an equation which using (7.9) is already rational in $\lambda$ and can be written

$$
\begin{aligned}
0 & =u_{0}^{4}\left(z_{0}\right)-2\left(\kappa_{+}^{2}+\kappa_{-}^{2}\right) u_{0}^{2}\left(z_{0}\right)+\left(\kappa_{+}^{2}-\kappa_{-}^{2}\right)^{2} \\
& =u_{0}^{4}\left(z_{0}\right)-2\left(u_{+}+u_{-}-2 \lambda\right) u_{0}^{2}\left(z_{0}\right)+\left(u_{+}-u_{-}\right)^{2} .
\end{aligned}
$$

From this we find in first-order approximation of the boundary conditions only one solution for $\lambda$

$$
\lambda=-\frac{u_{0}^{4}\left(z_{0}\right)-2\left(u_{+}+u_{-}\right) u_{0}^{2}\left(z_{0}\right)+\left(u_{+}-u_{-}\right)^{2}}{4 u_{0}^{2}\left(z_{0}\right)} .
$$

This solution is below the lower of the values of $u_{-}$and $u_{+}$, in considered case (7.4) lower than $u_{-}$that is also seen from the following representation of (7.15)

$$
\lambda-u_{-}=-\left(\frac{u_{0}^{2}\left(z_{0}\right)-\left(u_{+}-u_{-}\right)}{2 u_{0}\left(z_{0}\right)}\right)^{2} \leq 0 .
$$

According to (7.9) inserting (7.15) for $\lambda$ and choosing the signs of the arising square roots in the way that corresponding to (7.11) the moment $u_{0}\left(z_{0}\right)$ defined in (6.3) is non-positive this leads to

$$
\kappa_{+}=-\frac{u_{0}^{2}\left(z_{0}\right)+\left(u_{+}-u_{-}\right)}{2 u_{0}\left(z_{0}\right)} \geq \kappa_{-}=-\frac{u_{0}^{2}\left(z_{0}\right)-\left(u_{+}-u_{-}\right)}{2 u_{0}\left(z_{0}\right)} \geq 0,
$$


and this unambiguously is nearer to a potential well than to a potential barrier (next Section).

The bound state (excluding here its limiting case $\kappa_{+} \geq \kappa_{-}=0$ )

$$
\psi(z, \lambda)=A_{-} \mathrm{e}^{\kappa_{-} z} \theta\left(z_{0}-z\right)+A_{+} \mathrm{e}^{-\kappa_{+} z} \theta\left(z-z_{0}\right),
$$

is normalizable. As necessary and sufficient conditions for its presence follows from (7.11) and (7.17)

$$
u_{0}\left(z_{0}\right)<0, \quad u_{0}^{2}\left(z_{0}\right)>u_{+}-u_{-}>0 .
$$

The state (7.18) is concentrated to the neighborhood of the considered boundary $Z_{0}$. In this sense it is analogous to surface waves at a boundary between two media in electrodynamics only with the difference that in last case there is also a real propagation constant parallel to the surface.

\section{Asymptotically Equal Potentials and Bound States within a Potential Well}

We consider now the special case of equal asymptotically constant potentials $u_{-}=u_{+}$

$$
u_{-}=u_{+} \equiv u_{\infty} \text {, }
$$

from which follows that the first moment $u_{0}\left(z_{0}\right) \equiv u_{-}\left(z_{0}\right)+u_{+}\left(z_{0}\right)$ of the deviation of the real potential from the asymptotic potentials becomes independent on the reference point $z_{0}$, therefore

$$
u_{0}\left(z_{0}\right) \equiv \int_{-\infty}^{+\infty} \mathrm{d} z\left(u(z)-u_{\infty}\right)=u_{0}(0) .
$$

For $u_{0}(0)>0$ one may speak about a potential barrier and for $u_{0}(0)<0$ about a potential well but one may use these notions also in a stronger sense of $u(z)-u_{\infty}>0$ and of $u(z)-u_{\infty}<0$ only for all $z$.

All formulae derived in the last two Sections for the general case $u_{-} \neq u_{+}$remain true with the specialization (8.1) and only some formulae where $u_{-}-u_{+}$ stands in the denominator (such as (6.5) for a good choice of the reference point $z_{0}$ ) are no more applicable. For a "good" choice of the reference point $z_{0}$ one may choose instead that the first moment $u_{1}\left(z_{0}\right)$ defined now

$$
u_{1}\left(z_{0}\right) \equiv u_{-, 1}\left(z_{0}\right)+u_{+, 1}\left(z_{0}\right)=-\int_{-\infty}^{+\infty} \mathrm{d} z\left(u(z)-u_{\infty}\right)\left(z-z_{0}\right)=u_{1}(0)+z_{0} u_{0}(0),
$$

vanishes, from which follows for such choice

$$
0=u_{1}\left(z_{0}\right)=u_{1}(0)+z_{0} u_{0}(0), \Rightarrow z_{0}=-\frac{u_{1}(0)}{u_{0}(0)} .
$$

The normalizable bound states which vanish in infinity are now represented by $($ see $(7.8))$

$$
\begin{aligned}
& \psi_{-}(z, \lambda)=A_{-} \mathrm{e}^{+\kappa z}, \quad \psi_{+}(z, \lambda)=A_{+} \mathrm{e}^{-\kappa z}, \\
& \psi_{-}^{(1)}(z, \lambda)=\kappa A_{-} \mathrm{e}^{+\kappa z}, \quad \psi_{+}^{(1)}(z, \lambda)=-\kappa A_{+} \mathrm{e}^{-\kappa z} .
\end{aligned}
$$


For the only discrete state in considered first-order approximation of the boundary conditions follows then from (7.15)

$$
\lambda-u_{\infty}=-\frac{u_{0}^{2}(0)}{4}<0, \quad \kappa=\sqrt{u_{\infty}-\lambda}=-\frac{u_{0}(0)}{2}>0 .
$$

and the amplitude relations are

$$
A_{-} \mathrm{e}^{+\kappa z_{0}}=A_{+} \mathrm{e}^{-\kappa z_{0}} .
$$

The parameter $\lambda$ of the discrete state is in every case below the asymptotically constant potential $u_{\infty}$. All potential wells with the same area $u_{0}(0)$ below the asymptotic value $u_{\infty}$ (see also Figure A1 in Appendix B) are in first approximation equivalent

We now calculate the equation for normalizable discrete eigenstates in the third-order approximation of the boundary conditions. With the form of the solutions (8.5) follows from the boundary conditions in third-order approximation (4.22) two homogeneous equations which can be written in the following matrix form

$$
\mathrm{S}^{(2)}\left(\begin{array}{c}
A_{-} \mathrm{e}^{+\kappa z_{0}} \\
A_{+} \mathrm{e}^{-\kappa z_{0}}
\end{array}\right)=\left(\begin{array}{l}
0 \\
0
\end{array}\right)
$$

with the matrix $\left(\lambda=-\kappa^{2}\right)$

$$
\begin{gathered}
S^{(3)} \equiv\left(\begin{array}{c}
\left(1-u_{-, 1}\left(z_{0}\right)\right) \kappa+u_{-, 0}\left(z_{0}\right)+\left(u\left(z_{0}\right)-2 \lambda\right) u_{-, 2}\left(z_{0}\right) \\
1+u_{-, 1}\left(z_{0}\right)-2 u_{-, 2}\left(z_{0}\right) \kappa \\
\left(1+u_{+, 1}\left(z_{0}\right)\right) \kappa+u_{+, 0}\left(z_{0}\right)+\left(u\left(z_{0}\right)-2 \lambda\right) u_{+, 2}\left(z_{0}\right) \\
-1+u_{+, 1}\left(z_{0}\right)+2 u_{+, 2}\left(z_{0}\right) \kappa
\end{array}\right) .
\end{gathered}
$$

The condition of solvability of (8.8) is the vanishing of the determinant of $\mathrm{S}^{(3)}$ which leads to the following equation of third degree for $\kappa$

$$
\begin{aligned}
0= & 8 u_{-, 2}\left(z_{0}\right) u_{+, 2}\left(z_{0}\right) \kappa^{3}-4\left\{u_{-, 1}\left(z_{0}\right) u_{+, 2}\left(z_{0}\right)-u_{+, 1}\left(z_{0}\right) u_{-, 2}\left(z_{0}\right)\right\} \kappa^{2} \\
& -2\left\{1+u_{-, 1}\left(z_{0}\right) u_{+, 1}\left(z_{0}\right)-u_{-, 0}\left(z_{0}\right) u_{+, 2}\left(z_{0}\right)-u_{+, 0}\left(z_{0}\right) u_{-, 2}\left(z_{0}\right)\right. \\
& \left.-2 u\left(z_{0}\right) u_{-, 2}\left(z_{0}\right) u_{+, 2}\left(z_{0}\right)\right\} \kappa-\left\{u_{0}\left(z_{0}\right)-u_{-, 0}\left(z_{0}\right) u_{+, 1}\left(z_{0}\right)\right. \\
& \left.+u_{+, 0}\left(z_{0}\right) u_{-, 1}\left(z_{0}\right)+u\left(z_{0}\right)\left(u_{2}\left(z_{0}\right)+u_{-, 1}\left(z_{0}\right) u_{+, 2}\left(z_{0}\right)-u_{+, 1}\left(z_{0}\right) u_{-, 2}\left(z_{0}\right)\right)\right\}
\end{aligned}
$$

and for the amplitude relations we find from (8.8)

$$
\left(1+u_{-, 1}\left(z_{0}\right)-2 u_{-, 2}\left(z_{0}\right) \kappa\right) A_{-} \mathrm{e}^{\kappa z_{0}}=\left(1-u_{+, 1}\left(z_{0}\right)-2 u_{+, 2}\left(z_{0}\right) \kappa\right) A_{+} \mathrm{e}^{-\kappa z_{0}} .
$$

Our notations specialized to the case $(8.1)$ are $(m=0,1,2)$

$$
u_{m}\left(z_{0}\right) \equiv u_{-, m}\left(z_{0}\right)+u_{+, m}\left(z_{0}\right)=\frac{(-1)^{m}}{m !} \int_{-\infty}^{+\infty} \mathrm{d} z\left(u(z)-u_{\infty}\right)\left(z-z_{0}\right)^{m} .
$$

As a third-degree equation with real coefficients it may possess three real solutions or one real plus two complex-conjugate solutions where due to the smallness of the coefficients in front of $\kappa^{3}$ and of $\kappa^{2}$ the real solution (8.6) is only slightly modified and the new solutions are small from their modulus in 
comparison to (8.6).

\section{Favorable Form of Boundary Conditions for Layer Systems}

In case of a layer system with each single layer of the considered form it is favorable to represent the boundary conditions in a form where the amplitudes to each side of the layer stand on different sides that means

$$
\mathrm{S}_{-}\left(\begin{array}{c}
A_{-}^{\mathrm{i}} \mathrm{e}^{+\mathrm{i} k_{-} z_{0}} \\
A_{-}^{\mathrm{r}} \mathrm{e}^{-\mathrm{i} k_{-} z_{0}}
\end{array}\right)=\mathrm{S}_{+}\left(\begin{array}{c}
A_{+}^{\mathrm{r}} \mathrm{e}^{+\mathrm{i} k_{+} z_{0}} \\
A_{+}^{\mathrm{i}} \mathrm{e}^{-\mathrm{i} k_{+} z_{0}}
\end{array}\right)
$$

Then the output of one side of the transition layer may serve as input for the next transition layer which have to be in the asymptotically range because in other case we must add new boundary relations for the patch position of the two transition layers.

The resolution of (9.1) to the right-hand side of the amplitudes is

$$
\left(\begin{array}{l}
A_{+}^{\mathrm{r}} \mathrm{e}^{+\mathrm{i} k_{+} z_{0}} \\
A_{+}^{\mathrm{i}} \mathrm{e}^{-\mathrm{i} k_{+} z_{0}}
\end{array}\right)=\frac{\overline{\mathrm{S}_{+}} \mathrm{S}_{-}}{\left[\mathrm{S}_{+}\right]}\left(\begin{array}{c}
A_{-}^{\mathrm{i}} \mathrm{e}^{+\mathrm{i} k_{-} z_{0}} \\
A_{-}^{\mathrm{r}} \mathrm{e}^{-\mathrm{i} k_{-} z_{0}}
\end{array}\right) \equiv \mathrm{T}\left(\begin{array}{l}
A_{-}^{\mathrm{i}} \mathrm{e}^{+\mathrm{i} k_{-} z_{0}} \\
A_{-}^{\mathrm{r}} \mathrm{e}^{-\mathrm{i} k_{-} z_{0}}
\end{array}\right) \equiv\left(\begin{array}{cc}
T^{\mathrm{ri}} & T^{\mathrm{rr}} \\
T^{\mathrm{ii}} & T^{\mathrm{ir}}
\end{array}\right)\left(\begin{array}{l}
A_{-}^{\mathrm{i}} \mathrm{e}^{+\mathrm{i} k_{-} z_{0}} \\
A_{-}^{\mathrm{r}} \mathrm{e}^{-\mathrm{i} k_{-} z_{0}}
\end{array}\right) .
$$

In addition, it would be favorable to substitute in case of many layers when their connection is in the asymptotic ranges the lower indices “-” and "+" by specific notations of the involved layers to both sides of the transitions. The matrices $\mathrm{T} \equiv \frac{\overline{\mathrm{S}_{+} \mathrm{S}_{-}}}{\left[\mathrm{S}_{+}\right]}$we may call transmission matrices.

In second-order approximation we obtain for the matrices $\mathrm{S}_{+}$and $\mathrm{S}_{-}$under the same propositions (5.2) as before

$$
\begin{aligned}
& \mathrm{S}_{-}=\left(\begin{array}{cc}
k_{-}\left(1-u_{-, 1}\left(z_{0}\right)\right)-\mathrm{i} u_{-, 0}\left(z_{0}\right) & -k_{-}\left(1-u_{-, 1}\left(z_{0}\right)\right)-\mathrm{i} u_{-, 0}\left(z_{0}\right) \\
1+u_{-, 1}\left(z_{0}\right) & 1+u_{-, 1}\left(z_{0}\right)
\end{array}\right), \\
& \mathrm{S}_{+}=\left(\begin{array}{cc}
k_{+}\left(1+u_{+, 1}\left(z_{0}\right)\right)+\mathrm{i} u_{+, 0}\left(z_{0}\right) & -k_{+}\left(1+u_{+, 1}\left(z_{0}\right)\right)+\mathrm{i} u_{+, 0}\left(z_{0}\right) \\
1-u_{+, 1}\left(z_{0}\right) & 1-u_{+, 1}\left(z_{0}\right)
\end{array}\right) .
\end{aligned}
$$

The determinant $\left[\mathrm{S}_{+}\right]$of the matrix $\mathrm{S}_{+}$is

$$
\left[\mathrm{S}_{+}\right]=2\left(1-u_{+, 1}\left(z_{0}\right)\right)\left(1+u_{+, 1}\left(z_{0}\right)\right) k_{+} \text {. }
$$

Except trivial cases this determinant and the determinant $\left[\mathrm{S}_{-}\right]$of the matrix $\mathrm{S}_{\text {_ }}$ too cannot vanish and solutions which vanish only on one side of the transition layer do not exist. For the components of the transmission matrices $\mathrm{T}$ we calculate from (9.2)

$$
\begin{aligned}
{\left[\mathrm{S}_{+}\right] T^{\mathrm{ri}}=} & \left(1+u_{+, 1}\left(z_{0}\right) u_{-, 1}\left(z_{0}\right)\right)\left(k_{+}+k_{-}\right)+u_{1}\left(z_{0}\right)\left(k_{+}-k_{-}\right) \\
& -\mathrm{i}\left(u_{0}\left(z_{0}\right)+u_{+, 0}\left(z_{0}\right) u_{-, 1}\left(z_{0}\right)-u_{-, 0}\left(z_{0}\right) u_{+, 1}\left(z_{0}\right)\right), \\
{\left[\mathrm{S}_{+}\right] T^{\mathrm{rr}}=} & \left(1+u_{+, 1}\left(z_{0}\right) u_{-, 1}\left(z_{0}\right)\right)\left(k_{+}-k_{-}\right)+u_{1}\left(z_{0}\right)\left(k_{+}+k_{-}\right) \\
& -\mathrm{i}\left(u_{0}\left(z_{0}\right)+u_{+, 0}\left(z_{0}\right) u_{-, 1}\left(z_{0}\right)-u_{-, 0}\left(z_{0}\right) u_{+, 1}\left(z_{0}\right)\right),
\end{aligned}
$$




$$
\begin{aligned}
{\left[\mathrm{S}_{+}\right] T^{\mathrm{ii}}=} & \left(1+u_{+, 1}\left(z_{0}\right) u_{-, 1}\left(z_{0}\right)\right)\left(k_{+}-k_{-}\right)+u_{1}\left(z_{0}\right)\left(k_{+}+k_{-}\right) \\
& +\mathrm{i}\left(u_{0}\left(z_{0}\right)+u_{+, 0}\left(z_{0}\right) u_{-, 1}\left(z_{0}\right)-u_{-, 0}\left(z_{0}\right) u_{+, 1}\left(z_{0}\right)\right), \\
{\left[\mathrm{S}_{+}\right] T^{\mathrm{ir}}=} & \left(1+u_{+, 1}\left(z_{0}\right) u_{-, 1}\left(z_{0}\right)\right)\left(k_{+}+k_{-}\right)+u_{1}\left(z_{0}\right)\left(k_{+}-k_{-}\right) \\
& +\mathrm{i}\left(u_{0}\left(z_{0}\right)+u_{+, 0}\left(z_{0}\right) u_{-, 1}\left(z_{0}\right)-u_{-, 0}\left(z_{0}\right) u_{+, 1}\left(z_{0}\right)\right) .
\end{aligned}
$$

In the first-order approximation we neglect the moments $u_{+, 1}\left(z_{0}\right)$ and $u_{-, 1}\left(z_{0}\right)$ that we do not write down separately.

\section{Conclusions}

In this article, we have chosen the important Sturm-Liouville equation as one of the simplest ordinary second-order differential equations to demonstrate important aspects of the derivation of generalized boundary conditions by means of Generalized functions or, more especially, of step functions, delta functions and their derivatives. A countable infinite system of boundary conditions has been obtained and its first few low-order approximations are derived for this equation in case of sufficiently smooth potentials. It was necessary to choose two asymptotic "volume" potentials with known solutions of the corresponding Sturm-Liouville equation and an imaginary' boundary surface in the transition region between these asymptotic potentials. The information about the deviations of the real potential from the chosen asymptotic potentials on both sides of the "imaginary" boundary surface is involved in the boundary conditions in form of the moments of these deviations. The condition for the applicability of low-order approximations of the boundary conditions requires that the "effective" thickness of the transition region must be small in comparison to the characteristic lengths in alterations of the "volume" solutions in the transition region (wavelengths in the case of asymptotic constant potentials, for instance). Although this is intuitively clear, it is convenient to discuss this more precisely not in general abstract form but in connection with concrete potentials and problems. We applied it to reflection and refraction problems for asymptotically constant potentials.

The general problems of the derivation of boundary conditions by means of the theory of Generalized functions could be solved for the Sturm-Liouville equation. It seems that there are no principal difficulties to derive boundary conditions in an analogous way for other linear scalar or vectorial differential or integro-differential equations as was already done for electrodynamics. However, we do not want to conceal that in the case of nonlinear equations there are some new principal difficulties with the presented method.

\section{Acknowledgements}

Lately, I am able to express my great gratitude to Prof. G. Vojta (from Editorial Board of Ann. d. Physik in former GDR) who advocated the publication of my paper [12] against the rejection without a written report by the Chief Editor of Ann. d. Physik Prof. G. Richter from GDR who at that same time was also a 
Member of our Central Institute of Optics and Spectroscopy (ZOS). The arguments of G. Richter were unjustified and unacceptable (see my home page) ${ }^{5}$. Furthermore, in particular, I thank Dr. Th. Jung from "Zentralinstitut für Elektronenphysik" der "Akademie der Wissenschaften der DDR" who managed to give a colloquium lecture in mentioned Institute and also Prof. V.M. Agranovich from Moscow where during a one-month visit I could give a seminar lecture in his "Institute of Spectroscopy" nearby Moscow to the problems dealt with in [12]. In my former "Zentralinstitut für Optik und Spektroskopy” I never gave a lecture about these problems to avoid further trouble with some persons.

\section{Conflicts of Interest}

The author declares no conflicts of interest regarding the publication of this paper.

\section{References}

[1] Drude, P. (1889) Ueber Oberflächenschichten. Wiedemann's Annalen der Physik und Chemie, 36, I. Theil 532-560, II. Theil 865-897.

[2] Drude, P. (1912) Lehrbuch der Optik [Dritte Erweiterte Auflage]. Hirzel, Leipzig. ${ }^{6}$

[3] Mayer, H. (1950) Physik dünner Schichten. Wissenschaftliche Verlags-Gesellschaft, Stuttgart.

[4] Heavens, O.S. (1955) Optical Properties of Thin Films. Butterworths, London.

[5] Rozenberg, G.B. (1958) Optika tonkosloinykh pokrytiy [Optics of Thin-Layered Coverings]. Gosfizmatizdat, Moskva.

[6] Vašiček, A. (1969) Optics of Thin Films. North-Holland, Amsterdam.

[7] Agranovich, V.M. and Ginzburg, V.L. (1979) Kristallooptika s uchotom prostranstvennoi dispersii i teoriya eksitonov [Crystal Optics with Spatial Dispersion]. Nauka, Moskva.

[8] Bedeaux, D. and Vlieger, J. (1973) A Phenomenological Theory of the Dielectric Properties of Thin Films. Physica, 67, 55-73. https://doi.org/10.1016/0031-8914(73)90022-0

[9] Bedeaux, D. and Vlieger, J. (1974) A Statistical Theory of the Dielectric Properties of Thin Island Films: I. The Surface Material Coefficients. Physica, 73, 287-311. https://doi.org/10.1016/0031-8914(74)90002-0

[10] Timashev, S.F. and Krykin, A.M. (1976) Optical and Electrooptical Properties of the Interfaces of Media. Physica Status Solidi (b), 76, 67-76.

https://doi.org/10.1002/pssb.2220760106

[11] Timashev, S.F. and Krykin, A.M. (1979) Appendix to Osnovy ellipsometrii [Foundations of Ellipsometry]. Nauka, Novosibirsk.

\footnotetext{
${ }^{5} \mathrm{~A}$ later paper with application of the derived boundary condition from my published paper [12] onto reflection and refraction at the boundary between isotropic and optically uniaxial media was again rejected by $\mathrm{G}$. Richter with the same unacceptable arguments and is not published.

${ }^{6}$ I overtook my exemplar (a translation into Russian from Publishing House 'ONTI Leningrad', 1935) from my former colleague Randolf Fischer after his death from cancer in 1988 in the age of about 53 . The history of this exemplar before this I do not know. Inside I find handwritten "Iz knig B. Popova" (from the books of B. Popov). I do not know whether R. Fischer bought this book in the former Sovyet Union as second-hand book or did he directly overtake it from a Russian colleague.
} 
[12] Wünsche, A. (1980) Grenzbedingungen der Elektrodynamik für Medien mit räumlicher Dispersion und Übergangsschichten. Annalen der Physik, 37, 121-142. https://doi.org/10.1002/andp.19804920205

[13] Lekner, J. (2016) Theory of Reflection. Springer, Heidelberg.

[14] Landau, L.D. and Lifshits, Ye.M. (1982) Elektrodinamika sploshnykh sred [Electrodynamics of Continuous Media]. Nauka, Moskva.

[15] Levitan, B.M. and Sargsyan, I.S. (1970) Vvedenye v spektralnuyu teoriyu [Introduction to Spectral Theory]. Nauka, Moskva.

[16] Levitan, B.M. and Sargsyan, I.S. (1988) Operatory Shturma-Liuvilly [Sturm-Liouville Operators). Nauka, Moskva.

[17] Marchenko, V.A. (1977) Operatory Shturma-Liuvilla i yikh prilozhenya [SturmLiouville Operators and Their Application]. Naukova dumka, Kiev.

[18] Birman, M.S. and Faddeyev, L.D. (1972) Operatory kvantovoy mekhaniki [Operators of Quantum Mechanics]. Nauka, Moskva .

[19] Krein, S.G. (1972) Funktsional'nyi analiz [Functional Analysis]. 2nd Edition, Nauka, Moskva.

[20] Landau, L.D. and Lifshits, Ye.M. (1974) Kvantovaya mekhanika [Quantum Mechanics]. Nauka, Moskva.

[21] Morse, P.M. and Feshbach, H. (1953) Methods of Theoretical Physics, Part II. McGraw Hill, New York.

[22] Flügge, S. (1999) Practical Quantum Mechanics. Springer-Verlag, Berlin.

[23] Brekhovskikh, L.M. (1973) Volny v sloistyhk sredakh [Waves in Layered Media]. Nauka, Moskva.

[24] de Broglie, L. (1925) Recherches sur la théorie des Quanta. Annales de Physique, 10, 22-128. https://doi.org/10.1051/anphys/192510030022

[25] Gel'fand, I.M. and Shilov, G.Ye. (1959) Obobstchonnyje funktsii, I [Generalized Functions]. Vol. 1, Fizmatgiz, Moskva.

[26] Schwartz, L. (1961) Méthods Mathematiques pour les Science Physiques. Hermann, Paris.

[27] Vladimirov, V.S. (1971) Uravnyeniya matematicheskoi fiziki [Equations of Mathematical Physics]. Nauka, Moskva.

[28] Vilyenkin, N.Ya. (1972) Obobstchonnyje funktsii [Generalized Functions]. Nauka, Moskva.

[29] Bateman, H. and Erdélyi, A. (1954) Tables of Integral Transforms, Vol. II. McGrawHill, New York. 


\section{Appendix A. Multiplication of Delta Function and of Its Derivatives with Smooth Functions and Moment Series of Generalized Functions}

We derive in this Appendix some identities for generalized functions which we use in the main text. Generalized functions $f(x)$ of a function space $K^{*}$ are defined as linear continuous functionals over basis functions $\varphi(x)$ of a space $K$ of (sufficiently well-behaved, e.g., smooth, i.e., continuous and infinitely continuously differentiable) functions which we may write as generalization of the integral on the right-hand side in the relation [25] [26] [27] [28]

$$
(f, \varphi) \equiv \int_{-\infty}^{+\infty} \mathrm{d} x f(x) \varphi(x), \quad \lim _{k \rightarrow \infty}\left(f_{k}, \varphi\right)=(f, \varphi) .
$$

The delta function with real displacement parameter $x_{0}$ and their $n$-th derivatives $\delta^{(n)}\left(x-x_{0}\right)$ according to the rule of partial integration are defined by

$$
\int_{-\infty}^{+\infty} \mathrm{d} x \delta^{(n)}\left(x-x_{0}\right) \varphi(x)=(-1)^{n} \int_{-\infty}^{+\infty} \mathrm{d} x \delta\left(x-x_{0}\right) \varphi^{(n)}(x)=(-1)^{n} \varphi^{(n)}\left(x_{0}\right),
$$

with the important special cases for $\varphi(x)=1$

$$
\int_{-\infty}^{+\infty} \mathrm{d} x \delta^{(n)}\left(x-x_{0}\right)=\delta_{n, 0}=\left\{\begin{array}{ll}
1, & n=0 \\
0, & n \neq 0
\end{array},\right.
$$

showing that all "integrals" over genuine (i.e., $n \geq 1$ ) derivatives of the delta function taken over the real axis vanish. With possible (e.g., smooth) multiplication functions $\alpha(x)$ using the Leibniz rule for differentiation of a product one finds

$$
\begin{aligned}
& \int_{-\infty}^{+\infty} \mathrm{d} x \alpha(x) \delta^{(n)}\left(x-x_{0}\right) \varphi(x) \\
& =(-1)^{n} \int_{-\infty}^{+\infty} \mathrm{d} x \delta\left(x-x_{0}\right)(\alpha(x) \varphi(x))^{(n)} \\
& =(-1)^{n} \sum_{k=0}^{n} \frac{n !}{k !(n-k) !} \alpha^{(k)}\left(x_{0}\right) \varphi^{(n-k)}\left(x_{0}\right) \\
& =\sum_{k=0}^{n} \frac{(-1)^{k} n !}{k !(n-k) !} \alpha^{(k)}\left(x_{0}\right) \int_{-\infty}^{+\infty} \mathrm{d} x \delta^{(n-k)}\left(x-x_{0}\right) \varphi(x),
\end{aligned}
$$

and since this is correct for arbitrary basis functions $\varphi(x)$

$$
\alpha(x) \delta^{(n)}\left(x-x_{0}\right)=\sum_{k=0}^{n} \frac{(-1)^{k} n !}{k !(n-k) !} \alpha^{(k)}\left(x_{0}\right) \delta^{(n-k)}\left(x-x_{0}\right) .
$$

On the right-hand side of this identity we have only constant coefficients in front of the derivatives of the delta function $\delta\left(x-x_{0}\right)$.

We give two examples for (A.5). The first example with $\alpha(x)=x^{m}$ is

$$
\begin{aligned}
x^{m} \delta^{(n)}\left(x-x_{0}\right) & =\sum_{k=0}^{\{m, n\}} \frac{(-1)^{k} m ! n !}{k !(m-k) !(n-k) !} x_{0}^{m-k} \delta^{(n-k)}\left(x-x_{0}\right) \\
& =\mathrm{L}_{m, n}\left(x_{0}, \frac{\partial}{\partial x}\right) \delta\left(x-x_{0}\right),
\end{aligned}
$$

with the special case $x_{0}=0$ 


$$
x^{m} \delta^{(n)}(x)=\frac{(-1)^{m} n !}{(n-m) !} \delta^{(n-m)}(x)=\mathrm{L}_{m, n}\left(0, \frac{\partial}{\partial x}\right) \delta(x)
$$

In (A.6) $\mathrm{L}_{m, n}(x, y)$ denotes the Laguerre 2D polynomials defined by

$$
\begin{aligned}
\mathrm{L}_{m, n}(x, y) & \equiv \sum_{k=0}^{\{m, n\}} \frac{(-1)^{k} m ! n !}{k !(m-k) !(n-k) !} x^{m-k} y^{n-k} \\
& =(-1)^{n} n ! x^{m-n} \mathrm{~L}_{n}^{m-n}(x y)=(-1)^{m} m ! y^{n-m} \mathrm{~L}_{m}^{n-m}(x y),
\end{aligned}
$$

where $L_{n}^{v}(z)$ denotes the Generalized Laguerre polynomials or Laguerre-Sonin polynomials in standard notation.

A second example for illustration with $\alpha(x)=\exp \left(-\frac{x^{2}}{a^{2}}\right)$ is

$$
\begin{aligned}
& \exp \left(-\frac{x^{2}}{a^{2}}\right) \delta^{(n)}\left(x-x_{0}\right) \\
& =\exp \left(-\frac{x_{0}^{2}}{a^{2}}\right) \sum_{k=0}^{n} \frac{n !}{k !(n-k) !} \frac{1}{a^{k}} \mathrm{H}_{k}\left(\frac{x_{0}}{a}\right) \delta^{(n-k)}\left(x-x_{0}\right) \\
& =\exp \left(-\frac{x_{0}^{2}}{a^{2}}\right) \frac{1}{a^{n}} \mathrm{H}_{n}\left(\frac{x_{0}}{a}+\frac{a}{2} \frac{\partial}{\partial x}\right) \delta\left(x-x_{0}\right),
\end{aligned}
$$

with the special case $x_{0}=0$

$$
\exp \left(-\frac{x^{2}}{a^{2}}\right) \delta^{(n)}(x)=\sum_{l=0}^{\left[\frac{n}{2}\right]} \frac{(-1)^{l} n !}{l !(n-2 l) !} \frac{1}{a^{2 l}} \delta^{(n-2 l)}(x)=\frac{1}{a^{n}} \mathrm{H}_{n}\left(\frac{a}{2} \frac{\partial}{\partial x}\right) \delta(x),
$$

where $\mathrm{H}_{n}(z)$ denotes the Hermite polynomials

$$
\begin{aligned}
\mathrm{H}_{n}(z) & \equiv(-1)^{n} \exp \left(z^{2}\right) \frac{\partial^{n}}{\partial z^{n}} \exp \left(-z^{2}\right)=\exp \left(-\frac{1}{4} \frac{\partial^{2}}{\partial z^{2}}\right)(2 z)^{n} \\
& =\sum_{k=0}^{\left[\frac{n}{2}\right]} \frac{(-1)^{k} n !}{k !(n-2 k) !}(2 z)^{n-2 k},
\end{aligned}
$$

in different representations which can be used as alternative definitions.

An equation of the form

$$
0=\sum_{n=0}^{\infty} c_{n}\left(x_{0}\right) \delta^{(n)}\left(x-x_{0}\right)=c_{0}\left(x_{0}\right) \delta\left(x-x_{0}\right)+c_{1}\left(x_{0}\right) \delta^{(1)}\left(x-x_{0}\right)+\cdots,
$$

with coefficients $c_{n}\left(x_{0}\right)$ which are independent of the variable $x$ is only satisfied if all coefficients $c_{n}\left(x_{0}\right)$ vanish. This follows by multiplication of this equation with the functions $\left(x-x_{0}\right)^{m},(m=0,1,2, \cdots)$ using (A.7) and succeeding integration over the whole $x$-axis using (A.3) according to

$$
\begin{aligned}
0 & =\int_{-\infty}^{+\infty} \mathrm{d} x \sum_{n=0}^{\infty} c_{n}\left(x_{0}\right)\left(x-x_{0}\right)^{m} \delta^{(n)}\left(x-x_{0}\right) \\
& =\sum_{n=0}^{\infty} c_{n}\left(x_{0}\right) \frac{(-1)^{m} n !}{(n-m) !} \int_{-\infty}^{+\infty} \mathrm{d} x \delta^{(n-m)}\left(x-x_{0}\right) \\
& =(-1)^{m} m ! c_{m}\left(x_{0}\right), \Rightarrow c_{m}\left(x_{0}\right)=0, \quad(m=0,1,2, \cdots) .
\end{aligned}
$$


It means that the systems of functions $\left(x-x_{0}\right)^{m}$ and generalized functions $\delta^{n}\left(x-x_{0}\right)$ with arbitrary parameter $x_{0}$ are bi-orthogonal. For the derivation of boundary conditions for an equation one has to split the asymptotic parts and has to transform the remaining parts of the equation to the principal form (A.12) and find then the boundary condition from the vanishing of the coefficients $c_{m},(m=0,1,2, \cdots)$. From (A.13) follows with variation of the parameter $x_{0}$

$$
c_{n}\left(x_{0}\right)=\sum_{k=0}^{n} \frac{x_{0}^{k}}{k !} c_{n-k}(0) \text {. }
$$

This means that with variation of the parameter $x_{0}$ the whole system of boundary conditions transforms into each other. However, by truncation of the boundary conditions with finite numbers $n=N$ this full equivalence becomes an approximate equivalence and a "best" choice of the parameter $x_{0}$ becomes important.

We now generalize the preceding considerations to expansions of generalized functions which we call moment series because the coefficients of these expansions are determined by the moments of the function. For this purpose we first define the Fourier transform $\tilde{f}(k)$ of $f(x)$ which together with its inversion is given by

$$
\tilde{f}(u)=\int_{-\infty}^{+\infty} \mathrm{d} y f(y) \exp (-\mathrm{i} y u), \Leftrightarrow f(x)=\frac{1}{2 \pi} \int_{-\infty}^{+\infty} \mathrm{d} u \tilde{f}(u) \exp (\mathrm{i} u x) .
$$

Then we represent the function $f(x)$ as convolution (notation " $*$ ") of this function with the delta function and apply the displacement operator of the argument of a function as follows

$$
\begin{aligned}
f(x) & =f(x) * \delta(x)=\int_{-\infty}^{+\infty} \mathrm{d} y f(y) \delta(x-y) \\
& =\int_{-\infty}^{+\infty} \mathrm{d} y f(y) \exp \left(-y \frac{\partial}{\partial x}\right) \delta(x)=\tilde{f}\left(-\mathrm{i} \frac{\partial}{\partial x}\right) \delta(x),
\end{aligned}
$$

where we applied in last step the definition (A.15) of the Fourier transform. Making now expansions of the function $\delta(x-y)$ in a Taylor series with respect to powers of $y$ and $\tilde{f}(k)$ in a Taylor series at $k=0$ we find from (A.16)

$$
f(x)=\sum_{n=0}^{\infty} f_{n}(0) \delta^{(n)}(x)=\sum_{n=0}^{\infty} \frac{\tilde{f}^{(n)}(0)}{n !}\left(-\mathrm{i} \frac{\partial}{\partial x}\right)^{n} \delta(x),
$$

with the moments $f_{n}(0)$ of the function $f(x)$ defined by

$$
f_{n}(0) \equiv \frac{(-1)^{n}}{n !} \int_{-\infty}^{+\infty} \mathrm{d} y f(y) y^{n}=\frac{(-\mathrm{i})^{n}}{n !} \tilde{f}^{(n)}(0) .
$$

This shows that the moments of $f(x)$ are related to the derivatives of the Fourier transforms $\tilde{f}(u)$ at $x=0$. Conversely, the derivatives of the function $f(x)$ at $x_{0}=0$ are related to the moments of its Fourier transform $\tilde{f}(u)$ by

$$
f^{(n)}(0)=\frac{(-\mathrm{i})^{n}}{2 \pi} n ! \tilde{f}_{n}(0),
$$

with $\tilde{f}_{n}(0)$ defined by 


$$
\tilde{f}_{n}(0) \equiv \frac{(-1)^{n}}{n !} \int_{-\infty}^{+\infty} \mathrm{d} u \tilde{f}(u) u^{n}=2 \pi \frac{\mathrm{i}^{n}}{n !} f^{(n)}(0) .
$$

The relations (A.18) and (A.19) express a certain duality of the moments of a function and of the derivatives of its Fourier transforms at the coordinate origin. Upper indices in the form $f^{(n)}(x)$ mean in every case the $n$-th derivative of $f(x)$ and lower indices in the form $f_{n}\left(x_{0}\right)$ moments of a function to reference point $x_{0}^{7}$.

The convergence of moment series is weak convergence in the sense of the convergence of the sequence of partial sums as linear functionals over the space of basis functions such as written in (A.1). The linear continuous functionals over basis functions $\varphi(x)$ can be transformed by Fourier transformation (A.15) in linear continuous functionals over the space of Fourier transforms to the basis functions of the space $\mathcal{D}$ usually denoted by $\mathcal{Z}$ as follows

$$
\begin{aligned}
(f, \varphi) & \equiv \int_{-\infty}^{+\infty} \mathrm{d} x f(x) \varphi(x) \\
& =\int_{-\infty}^{+\infty} \mathrm{d} x \frac{1}{2 \pi} \int_{-\infty}^{+\infty} \tilde{f}(u) \mathrm{e}^{\mathrm{i} u x} \varphi(x) \\
& =\int_{-\infty}^{+\infty} \mathrm{d} u \tilde{f}(u) \frac{1}{2 \pi} \int_{-\infty}^{+\infty} \mathrm{d} x \varphi(x) \mathrm{e}^{\mathrm{i} x u} \\
& =\frac{1}{2 \pi} \int_{-\infty}^{+\infty} \mathrm{d} u \tilde{f}(u) \tilde{\varphi}(-u) \equiv \frac{1}{2 \pi}\left(\tilde{f}, \tilde{\varphi}^{*}\right),
\end{aligned}
$$

where we changed the order of integrations. The obtained relation is the Parseval theorem.

The expansion (A.17) can be generalized to an arbitrary reference point $x_{0}$ instead of $x_{0}=0$ and instead of (A.16) we have

$$
\begin{aligned}
f(x) & =\int_{-\infty}^{+\infty} \mathrm{d} y f(y) \delta\left(x-x_{0}+x_{0}-y\right) \\
& =\int_{-\infty}^{+\infty} \mathrm{d} y f(y) \exp \left(\left(x_{0}-y\right) \frac{\partial}{\partial x}\right) \delta\left(x-x_{0}\right) \\
& =\exp \left(x_{0} \frac{\partial}{\partial x}\right) \tilde{f}\left(-\mathrm{i} \frac{\partial}{\partial x}\right) \delta\left(x-x_{0}\right) .
\end{aligned}
$$

The operators $\exp \left(x_{0} \frac{\partial}{\partial x}\right)$ and $\tilde{f}\left(-\mathrm{i} \frac{\partial}{\partial x}\right)$ commute and using this it is easy to return to the form (A.16) which also follows as special case setting $x_{0}=0$. From (A.22) follows

$$
f(x)=\sum_{n=0}^{\infty} f_{n}\left(x_{0}\right) \delta^{(n)}\left(x-x_{0}\right),
$$

with the moments $f_{n}\left(x_{0}\right)$ determined by

$$
\begin{aligned}
f_{n}\left(x_{0}\right) & \equiv \frac{(-1)^{n}}{n !} \int_{-\infty}^{+\infty} \mathrm{d} y f(y)\left(y-x_{0}\right)^{n}=\sum_{k=0}^{n} \frac{x_{0}^{k}}{k !} f_{n-k}(0) \\
& =\sum_{k=0}^{n} \frac{(-\mathrm{i})^{n-k}}{k !(n-k) !} x_{0}^{k} \tilde{f}^{(n-k)}(0)=\frac{1}{n !}\left\{\left(x_{0}-\mathrm{i} \frac{\partial}{\partial u}\right)^{n} \tilde{f}(u)\right\}_{u=0},
\end{aligned}
$$

${ }^{7}$ In comparison to my paper [12] from 1980 I changed here slightly the principles of index notations. 
where the relation of the moments at reference point $x=x_{0}$ to the moments at reference point $x=0$ is given. The zeroth moment $f_{0}\left(x_{0}\right)$ does not depend on the reference point $x_{0}$

$$
f_{0}\left(x_{0}\right)=f_{0}(0)=\tilde{f}(0)=\int_{-\infty}^{+\infty} \mathrm{d} y f(y),
$$

and, therefore, is "absolute". Furthermore, this formula shows how the moments to the reference points $x_{0}$ of a function can be expressed in simple way by the Fourier transform of this function.

For example, for a "normalized" Gaussian function together with its Fourier transform given by

$$
f(x)=\frac{1}{\sqrt{\pi a^{2}}} \exp \left(-\frac{x^{2}}{a^{2}}\right), \Leftrightarrow \tilde{f}(u)=\exp \left(-\frac{a^{2} u^{2}}{4}\right),
$$

we find the following moment series

$$
\frac{1}{\sqrt{\pi a^{2}}} \exp \left(-\frac{x^{2}}{a^{2}}\right)=\exp \left(\frac{a^{2}}{4} \frac{\partial^{2}}{\partial x^{2}}\right) \delta(x)=\sum_{m=0}^{\infty} \frac{a^{2 m}}{m ! 2^{2 m}} \delta^{(2 m)}(x),
$$

with the moments $f_{n}(0)$ determined by

$$
f_{2 m}(0)=\frac{a^{2 m}}{m ! 2^{2 m}}, \quad f_{2 m+1}(0)=0, \quad(m=0,1,2, \cdots) .
$$

Due to symmetry of the Gaussian function the odd moments are vanishing with respect to the reference point $x_{0}=0$ but not with respect to an arbitrary reference point $x_{0}$. Further examples for moment series of symmetric functions one may find in Appendix B.

As special cases we consider now the moment series for functions which are non-vanishing only to the left or to right of a reference point $x=x_{0}$.

For functions $f_{-}(x)$ which are non-vanishing only for variable $x<x_{0}$

$$
f_{-}(x) \equiv f(x) \theta\left(x_{0}-x\right)=\left\{\begin{array}{ll}
f(x), & x<x_{0} \\
0, & x>x_{0}
\end{array},\right.
$$

we find the moment series to reference point $x=x_{0}$

$$
f_{-}(x)=\sum_{n=0}^{\infty} f_{-, n}\left(x_{0}\right) \delta^{(n)}\left(x-x_{0}\right), \quad f_{-}\left(x_{0}+y\right)=\sum_{n=0}^{\infty} f_{-, n}\left(x_{0}\right) \delta^{(n)}(y),
$$

with the moments

$$
\begin{aligned}
f_{-, n}\left(x_{0}\right) & \equiv \frac{(-1)^{n}}{n !} \int_{-\infty}^{0} \mathrm{~d} y f\left(x_{0}+y\right) y^{n}=\frac{1}{n !} \int_{-\infty}^{x_{0}} \mathrm{~d} x f(x) \underbrace{\left(x_{0}-x\right)^{n}}_{\geq 0} \\
& =\int_{-\infty}^{x_{0}} \mathrm{~d} x_{n} \int_{-\infty}^{x_{n}} \mathrm{~d} x_{n-1} \cdots \int_{-\infty}^{x_{2}} \mathrm{~d} x_{1} \int_{-\infty}^{x_{1}} \mathrm{~d} x f(x),
\end{aligned}
$$

given together with an equivalent multiple integral.

For functions $f_{+}(x)$ which are non-vanishing only for variable $x>x_{0}$

$$
f_{+}(x) \equiv f(x) \theta\left(x-x_{0}\right)=\left\{\begin{array}{ll}
f(x), & x>x_{0} \\
0, & x<x_{0}
\end{array},\right.
$$


we find the moment series to reference point $x=x_{0}$

$$
f_{+}(x)=\sum_{n=0}^{\infty} f_{+, n}\left(x_{0}\right) \delta^{(n)}\left(x-x_{0}\right), \quad f_{+}\left(x_{0}+y\right)=\sum_{n=0}^{\infty} f_{+, n}\left(x_{0}\right) \delta^{(n)}(y),
$$

with the moments

$$
\begin{aligned}
f_{+, n}\left(x_{0}\right) & \equiv \frac{(-1)^{n}}{n !} \int_{0}^{+\infty} \mathrm{d} y f\left(x_{0}+y\right) y^{n}=\frac{(-1)^{n}}{n !} \int_{x_{0}}^{+\infty} \mathrm{d} x f(x) \underbrace{\left(x-x_{0}\right)^{n}}_{\geq 0} \\
& =(-1)^{n} \int_{x_{0}}^{+\infty} \mathrm{d} x_{n} \int_{x_{n}}^{+\infty} \mathrm{d} x_{n-1} \cdots \int_{x_{2}}^{+\infty} \mathrm{d} x_{1} \int_{x_{1}}^{+\infty} \mathrm{d} x f(x),
\end{aligned}
$$

given again together with an equivalent multiple integral.

The sum of the moments $f_{-, n}\left(x_{0}\right)$ and $f_{+, n}\left(x_{0}\right)$ is

$$
f(x)=f_{-}(x)+f_{+}(x), \quad f_{-, n}\left(x_{0}\right)+f_{+, n}\left(x_{0}\right)=f_{n}\left(x_{0}\right),
$$

to the moment series (A.23) with the moments determined by (A.24).

It should be mentioned here that the identities in the formulae (A.31) and (A.34) are special cases of the more general identity

$$
\begin{aligned}
f^{(-(n+1))}(a ; y) & \equiv \int_{a}^{y} \mathrm{~d} x_{n} \int_{a}^{x_{n}} \mathrm{~d} x_{n-1} \cdots \int_{a}^{x_{2}} \mathrm{~d} x_{1} \int_{a}^{x_{1}} \mathrm{~d} x f(x) \\
& =\frac{1}{n !} \int_{a}^{y} \mathrm{~d} x f(x)(y-x)^{n}
\end{aligned}
$$

with arbitrary parameter $a$ that can be proved by complete induction with reordering of the arising double integral and accomplishing then the inner integral. With the choice $a=0$ and $n \rightarrow \mu-1$ as an arbitrary real number it is often taken as starting point for the introduction of " $\mu$-fold" fractional integration by the convolution integral on the right-hand side which in our written case corresponds to possible $(n+1)$-fold integration of the function $f(x)$ (e.g., [29], Chap. XIII).

The moment series (A.23) can be made also for functions $f(x)$ which are not smooth and even not continuous and, roughly speaking, they are well defined if only all moments exist. The approximate reconstruction of the function $f(x)$ from the moment series can be obtained from the limits of the linear functionals $\left(f_{k}, \varphi\right)$ when $f_{k}$ denotes the truncated moment series up to the term with the $k$-th moment (see (A.1)). As basis functions $\varphi(x)$ it seems to be sufficient to take, for example, all displaced normalized Gaussian functions $\frac{1}{\sqrt{\pi \varepsilon^{2}}} \exp \left(-\frac{\left(x-x^{\prime}\right)^{2}}{\varepsilon^{2}}\right)$ with the limit $\varepsilon \rightarrow 0$. However also the Fourier transforms $\tilde{f}(u)$ are appropriate if their Taylor series may be summed up and if then the inverse Fourier transformation $f(x)$ to $\tilde{f}(u)$ is calculable.

\section{Appendix B. Examples of Potential Barriers and Potential Wells and of Transition Functions with Their Characteris- tics by Generalized Functions}

In this Appendix we consider for illustration some examples of functions $f(x)$ 
which are appropriate as potential barriers or potential wells together with their Fourier transforms $\tilde{f}(u)$ (notations as in (A.15)) and their moment series and, furthermore, functions which are appropriate to simulate transitions between two potentials for which the Sturm-Liouville equation can be exactly solved (e.g., constant potentials).

In Figure A1 we present six from seven explicitly given symmetric functions $f_{v}(x),(v=1, \cdots, 7)$ which are appropriate as potential barriers and, negatively taken, as potential wells

$$
\begin{aligned}
& \text { (1): } f_{1}(x)=\theta\left(1-\frac{2|x|}{a}\right), \quad \tilde{f}_{1}(u)=\frac{2}{u} \sin \left(\frac{a}{2} u\right), \\
& \text { (2): } f_{2}(x)=\sqrt{1-\left(\frac{\pi x}{2 a}\right)^{2}} \theta\left(1-\frac{\pi|x|}{2 a}\right), \quad \tilde{f}_{2}(u)=\frac{\pi}{u} \mathrm{~J}_{1}\left(\frac{2 a}{\pi} u\right), \\
& \text { (3): } f_{3}(x)=\left(1-\frac{|x|}{a}\right) \theta\left(1-\frac{|x|}{a}\right), \quad \tilde{f}_{3}(u)=a\left(\frac{\sin \left(\frac{a}{2} u\right)}{\frac{a}{2} u}\right) \\
& \text { (4): } f_{4}(x)=\exp \left(-\frac{2|x|}{a}\right), \quad \tilde{f}_{4}(u)=\frac{a}{1+\left(\frac{a}{2} u\right)^{2}}
\end{aligned}
$$
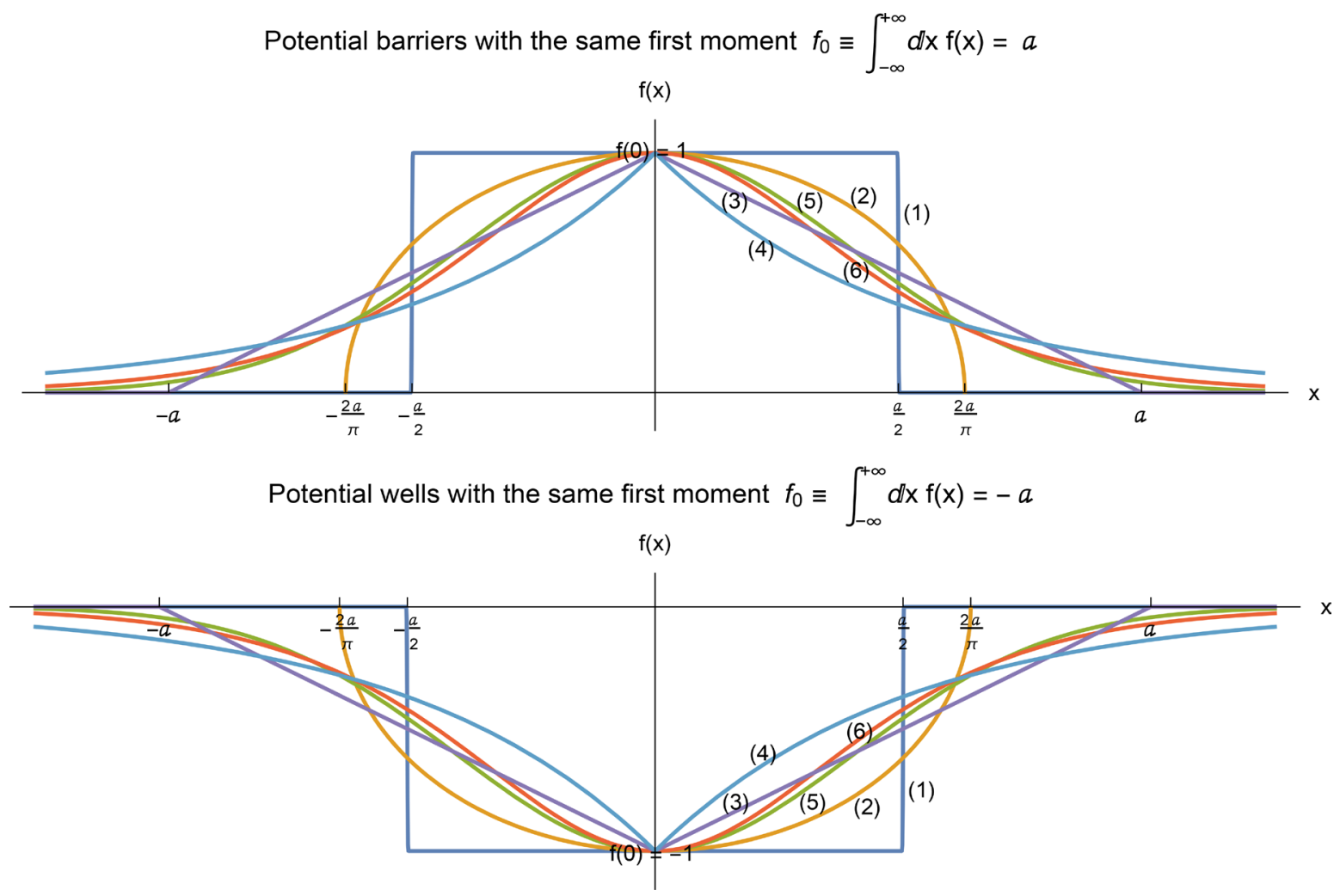

Figure A1. Examples for symmetric potential barriers and potential wells. The potential functions (5) and (6) and also (6) and (7) are very similar in some parts within the shown interval and it is difficult to distinguish them in the figure and therefore (7) is not drawn. The potential wells belong to the negatively taken functions $f(x)$. 
(5): $f_{5}(x)=\exp \left(-\frac{\pi x^{2}}{a^{2}}\right), \quad \tilde{f}_{5}(u)=a \exp \left(-\frac{a^{2}}{4 \pi} u^{2}\right)$,

(6): $f_{6}(x)=\frac{1}{\operatorname{ch}^{2}\left(\frac{2 x}{a}\right)}, \quad \tilde{f}_{6}(u)=a \frac{\pi a u}{4 \operatorname{sh}\left(\frac{\pi a}{4} u\right)}$,

(7): $f_{7}(x)=\frac{1}{\operatorname{ch}\left(\frac{\pi x}{a}\right)}, \quad \tilde{f}_{7}(u)=\frac{a}{\operatorname{ch}\left(\frac{a}{2} u\right)}$.

Their first three initial terms of the moment series are

(1): $f_{1}(x)=a\left\{\delta(x)+\frac{a^{2}}{24} \delta^{(2)}(x)+\frac{a^{4}}{1920} \delta^{(4)}(x)+\cdots\right\}$,

(2): $f_{2}(x)=a\left\{\delta(x)+\frac{a^{2}}{2 \pi^{2}} \delta^{(2)}(x)+\frac{a^{4}}{12 \pi^{4}} \delta^{(4)}(x)+\cdots\right\}$,

(3): $f_{3}(x)=a\left\{\delta(x)+\frac{a^{2}}{12} \delta^{(2)}(x)+\frac{a^{4}}{360} \delta^{(4)}(x)+\cdots\right\}$,

(4): $f_{4}(x)=a\left\{\delta(x)+\frac{a^{2}}{4} \delta^{(2)}(x)+\frac{a^{4}}{16} \delta^{(4)}(x)+\frac{a^{6}}{64} \delta^{(6)}(x)+\cdots\right\}$,

(5): $f_{5}(x)=a\left\{\delta(x)+\frac{a^{2}}{4 \pi} \delta^{(2)}(x)+\frac{a^{4}}{32 \pi^{2}} \delta^{(4)}(x)+\cdots\right\}$,

(6): $f_{6}(x)=a\left\{\delta(x)+\frac{\pi^{2} a^{2}}{96} \delta^{(2)}(x)+\frac{7 \pi^{4} a^{4}}{92160} \delta^{(4)}(x)+\cdots\right\}$,

(7): $f_{7}(x)=a\left\{\delta(x)+\frac{a^{2}}{8} \delta^{(2)}(x)+\frac{5 a^{4}}{384} \delta^{(4)}(x)+\cdots\right\}$.

All considered examples of functions $f_{v}(x)$ possess analytic Fourier transforms $\tilde{f}_{v}(u)$ and, therefore, well-defined moment series.

We now consider transition functions. Examples for transition functions can be constructed from the potential functions $f_{v}(x)$ in (B.2) by integration in the following way

$$
F_{v}(x)=\frac{1}{a} \int_{0}^{x} \mathrm{~d} y f_{v}(y), \quad F_{v}^{(1)}(x)=\frac{f_{v}(x)}{a}, \quad F_{v}(\mp \infty)=\mp \frac{1}{2} .
$$

Since we have chosen $\int_{-\infty}^{+\infty} \mathrm{d} x f(x)=a$ as the zeroth moment of the function $f(x)$ we obtain by (B.3) a transition step of the amount $F(+\infty)-F(-\infty)=1$ that means a unit step and an increase in the center $x=0$ of $F^{(1)}(0)=\frac{1}{a}$. For the functions in (B.2) these are the functions

$$
\text { (1): } F_{1}(x)= \begin{cases}-\frac{1}{2}, & x \leq-\frac{a}{2} \\ \frac{x}{a}, & -\frac{a}{2}<x<+\frac{a}{2}, \\ +\frac{1}{2}, & +\frac{a}{2}<x\end{cases}
$$


(2): $F_{2}(x)= \begin{cases}-\frac{1}{2}, & x \leq-\frac{2 a}{\pi} \\ \frac{x}{2 a} \sqrt{1-\left(\frac{\pi x}{2 a}\right)^{2}}+\frac{1}{\pi} \arcsin \left(\frac{\pi x}{2 a}\right), & -\frac{2 a}{\pi}<x<+\frac{2 a}{\pi}, \\ +\frac{1}{2}, & +\frac{2 a}{\pi}<x\end{cases}$

(3): $F_{3}(x)= \begin{cases}-\frac{1}{2}, & x \leq-a \\ \frac{x}{a}\left(1-\frac{|x|}{2 a}\right), & -a<x<+a, \\ +\frac{1}{2}, & +a<x\end{cases}$

(4): $F_{4}(x)=\frac{1}{2}\left(1-\exp \left(-\frac{2|x|}{a}\right)\right) \frac{x}{|x|}$,

(5): $F_{5}(x)=\frac{1}{2} \operatorname{erf}\left(\frac{\pi x}{2 a}\right)$,

(6): $F_{6}(x)=\frac{1}{2} \operatorname{th}\left(\frac{2 x}{a}\right)$,

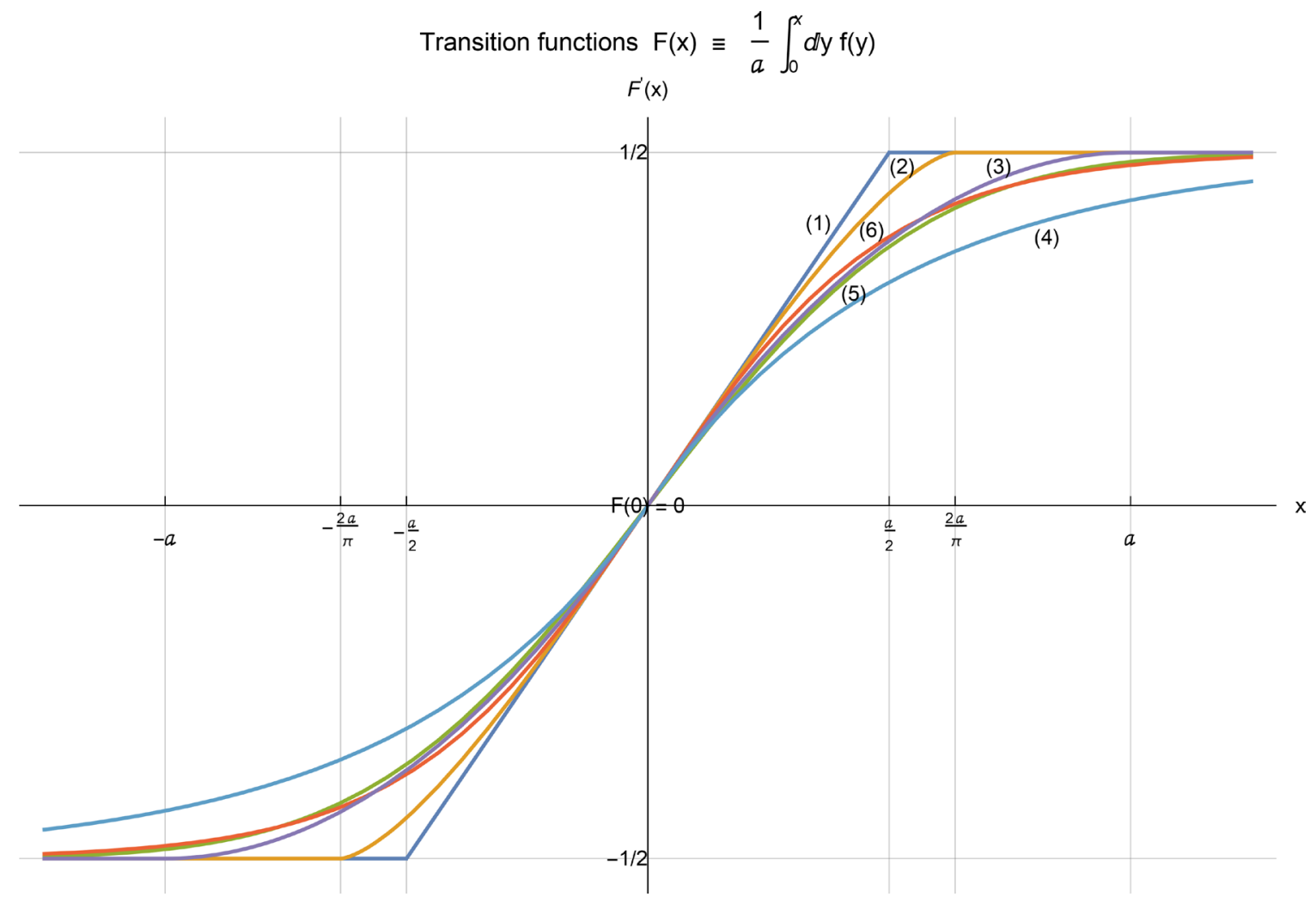

Figure A2. Examples for antisymmetric transition functions. The transition step is here normalized to unity $F(+\infty)-F(-\infty)=1$ and the increase in the center of the transition is equal to $F^{(1)}(0)=\frac{1}{a}$. Some of the transition functions, for example, (5) and (6) are very similar in some parts and are hardly to distinguish in this figure and (7) is also very near to (5) and (6) and therefore not drawn. 


$$
\text { (7): } F_{7}(x)=\frac{2}{\pi} \operatorname{arctg}\left(\operatorname{th}\left(\frac{\pi x}{2 a}\right)\right) \text {, }
$$

shown in Figure A2. In the first approximation of the boundary conditions all these transition functions are equivalent and only in higher approximations they provide differences in the results. 